\title{
NONLOCAL PERIMETER, CURVATURE AND MINIMAL SURFACES FOR MEASURABLE SETS
}

\author{
JOSÉ M. MAZÓN, JULIO D. ROSSI AND JULIÁN TOLEDO
}

\begin{abstract}
In this paper, we study the nonlocal perimeter associated with a nonnegative radial kernel $J: \mathbb{R}^{N} \rightarrow \mathbb{R}$, compactly supported, verifying $\int_{\mathbb{R}^{N}} J(z) d z=1$. The nonlocal perimeter studied here is given by the interactions (measured in terms of the kernel $J$ ) of particles from the outside of a measurable set $E$ with particles from the inside, that is,
\end{abstract}

$$
P_{J}(E):=\int_{E}\left(\int_{\mathbb{R}^{N} \backslash E} J(x-y) d y\right) d x .
$$

We prove that an isoperimetric inequality holds and that, when the kernel $J$ is appropriately rescaled, the nonlocal perimeter converges to the classical local perimeter. Associated with the kernel $J$ and the previous definition of perimeter we can consider minimal surfaces. In connexion with minimal surfaces we introduce the concept of $J$-mean curvature at a point $x$, and we show that again under rescaling we can recover the usual notion of mean curvature. In addition, we study the analogous to a Cheeger set in this nonlocal context and show that a set $\Omega$ is $J$-calibrable $(\Omega$ is a $J$-Cheeger set of itself) if and only if there exists $\tau$ such that $\tau(x)=1$ if $x \in \Omega$ satisfying $-\lambda_{\Omega}^{J} \tau \in \Delta_{1}^{J} \chi_{\Omega}$, here $\lambda_{\Omega}^{J}$ is the $J$-Cheeger constant $\lambda_{\Omega}^{J}=\frac{P_{J}(\Omega)}{|\Omega|}$ and, $\Delta_{1}^{J}$ is given, formally, by

$$
\Delta_{1}^{J} u(x)=\int_{\mathbb{R}^{N}} J(x-y) \frac{u(y)-u(x)}{|u(y)-u(x)|} d y .
$$

Moreover, we also provide a result on $J$-calibrable sets and the nonlocal $J$-mean curvature that says that a $J$-calibrable set can not include points with large curvature. Concerning examples, we show that balls are $J$-calibrable for kernels $J$ that are radially nonincresing, while stadiums are $J$-calibrable when they are small but they are not when they are large.

\section{INTRODUCTION}

Our main goal in this paper is to define a notion of perimeter, curvature and minimal surfaces for measurable sets without assuming any regularity condition on the boundary of the set. We also want our approach to be compatible with the usual notions of perimeter and curvature in the sense that we recover these usual quantities when we introduce a scaling parameter in our context that goes to zero.

Let $J: \mathbb{R}^{N} \rightarrow[0,+\infty[$ be a measurable, nonnegative and radially symmetric function verifying $\int_{\mathbb{R}^{N}} J(z) d z=1$. Associated with this function $J$, we introduce a nonlocal version of the usual perimeter of a set: let $E \subset \mathbb{R}^{N}$ be a measurable set, the nonlocal $J$-perimeter of $E$ is defined by

$$
P_{J}(E):=\int_{E}\left(\int_{\mathbb{R}^{N} \backslash E} J(x-y) d y\right) d x .
$$

Key words and phrases. Sets of finite perimeter, nonlocal operators, Cheeger sets, Calibrable sets. 2010 Mathematics Subject Classification: 45C99, 28A75, 49Q05. 
It is easy to see that

$$
P_{J}(E)=\frac{1}{2} \int_{\mathbb{R}^{N}} \int_{\mathbb{R}^{N}} J(x-y)\left|\chi_{E}(y)-\chi_{E}(x)\right| d x d y .
$$

Also, if $|E|<+\infty$, we have

$$
P_{J}(E)=|E|-\int_{E} \int_{E} J(x-y) d y d x
$$

This definition of perimeter is nonlocal in the sense that it is determined by the behaviour of $E$ in a neighbourhood of the boundary $\partial E$. The quantity $P_{J}(E)$ measures the interaction between points of $E$ and $\mathbb{R}^{N} \backslash E$ via the interaction density $J(x-y)$. For $J$ compactly supported in a ball $\bar{B}_{r}(0)$, the interaction is possible when the points $x \in E$ and $y \in \mathbb{R}^{N} \backslash E$ are both close to the boundary $\partial E$ :

$$
P_{J}(E)=\int_{\left\{x \in E: d\left(x, \mathbb{R}^{N} \backslash E\right)<r\right\}} \int_{\left\{y \in \mathbb{R}^{N} \backslash E: d(y, E)<r\right\}} J(x-y) d y d x .
$$

Since the kernel is not singular the concept of perimeter is well defined for every measurable set and is finite for every set with finite measure.

For the nonlocal perimeter there is an isoperimetric inequality when $J$ is radially nonincreasing. For every set $E$ with finite measure it holds that

$$
\frac{P_{J}(E)}{|E|} \geq \frac{P_{J}\left(B_{r}\right)}{\left|B_{r}\right|}
$$

where $B_{r}$ is a ball with the same measure as $E,\left|B_{r}\right|=|E|$ (here and in what follows we denote by $|E|$ the Lebesgue measure of a set $E$ in $\mathbb{R}^{N}$ ).

Associated with a kernel $J$ we define the space

$$
B V_{J}\left(\mathbb{R}^{N}\right):=\left\{u: \mathbb{R}^{N} \rightarrow \mathbb{R} \text { measurable }: \int_{\mathbb{R}^{N}} \int_{\mathbb{R}^{N}} J(x-y)|u(y)-u(x)| d x d y<\infty\right\} .
$$

We have that $L^{1}\left(\mathbb{R}^{N}\right) \subset B V_{J}\left(\mathbb{R}^{N}\right)$. For $u \in B V_{J}\left(\mathbb{R}^{N}\right)$, we also define the functional

$$
\mathcal{F}_{J}(u):=\frac{1}{2} \int_{\mathbb{R}^{N}} \int_{\mathbb{R}^{N}} J(x-y)|u(y)-u(x)| d x d y .
$$

This functional is the nonlocal analog of the energy functional associated with the total variation. In what follows we denote by $B V\left(\mathbb{R}^{N}\right)$ the set of functions of bounded total variation, i.e.,

$$
B V\left(\mathbb{R}^{N}\right):=\left\{u \in L^{1}\left(\mathbb{R}^{N}\right):|D u|\left(\mathbb{R}^{N}\right)<\infty\right\},
$$

where $|D u|$ is the total variation of the distributional gradient of $u$ ([5], [27]). If $\mathcal{F}$ is the energy functional associated with the total variation, i.e.,

$$
\mathcal{F}(u):=\int_{\mathbb{R}^{N}}|D u|, \quad u \in B V\left(\mathbb{R}^{N}\right),
$$

then a measurable set $E \subset \mathbb{R}^{N}$ has finite perimeter if $\chi_{E} \in B V\left(\mathbb{R}^{N}\right)$. The perimeter of $E$ is defined as

$$
\operatorname{Per}(E):=\int_{\mathbb{R}^{N}}\left|D \chi_{E}\right|
$$

Note that the nonlocal perimeter $P_{J}(E)$, written in the form (1.1), can be seen as the nonlocal version of $\operatorname{Per}(E)$.

The notion of nonlocal perimeter can be localized to a bounded open set $\Omega \subset \mathbb{R}^{N}$ by setting

$$
P_{J}(E, \Omega):=\int_{E} \int_{\mathbb{R}^{N} \backslash E} J(x-y) d y d x-\int_{E \backslash \Omega} \int_{\mathbb{R}^{N} \backslash(E \cup \Omega)} J(x-y) d y d x .
$$


If we define the nonlocal interaction between two measurable sets $A$ and $B$ of $\mathbb{R}^{N}$ as

$$
L_{J}(A, B):=\int_{A}\left(\int_{B} J(x-y) d y\right) d x
$$

(which is equatl to $L_{J}(B, A)$ by the symmetry of $J$ ), then we can rewrite the nonlocal perimeter as

$$
P_{J}(E)=L_{J}\left(E, \mathbb{R}^{N} \backslash E\right),
$$

and the localized nonlocal perimeter as

$$
P_{J}(E, \Omega)=L_{J}\left(E, \mathbb{R}^{N} \backslash E\right)-L_{J}\left(E \backslash \Omega, \mathbb{R}^{N} \backslash(E \cup \Omega)\right)=L_{J}\left(\Omega \cap E, \mathbb{R}^{N} \backslash E\right)+L_{J}(E \backslash \Omega, \Omega \backslash E) .
$$

Observe that, if we assume that in $B$ there is an homogeneous population (we assume density 1 of the population inside $B$ ) and $J(x-y)$ is the probability that an individual jumps from $y$ to $x$, then $L_{J}(A, B)$ is the total amount of individuals that arrives to $A$ from $B$. Note that one can think all this the other way arround, if we assume that in $A$ there is a population with density 1 , then $L_{J}(A, B)$ is the amount of individuals that goes from $A$ to $B$. Then we can remark that the individuals that go from $\mathbb{R}^{N} \backslash E$ to $E$ travel across the boundary of the set $E$, and, therefore, ours is a natural way of defining the perimeter of this set; we are counting the total flux of individuals that crosses the boundary when they go from $\mathbb{R}^{N} \backslash E$ to $E$.

For singular kernels, that is, for kernels of the form $J(\xi)=\frac{1}{|\xi|^{N+s}}$ the concept of nonlocal perimeter was introduced in [18] (see also the pioneering works [35], [36], where some functionals of this type were analyzed in connection with fractal dimensions). For $0<s<1$, the $s$-perimeter of $E \subset \mathbb{R}^{N}$ is defined (formally) as

$$
\operatorname{Per}_{s}(E):=\int_{E} \int_{\mathbb{R}^{N} \backslash E} \frac{1}{|x-y|^{N+s}} d x d y .
$$

The usual notion of perimeter is recovered by the limit

$$
\lim _{s \rightarrow 1}(1-s) \operatorname{Per}_{s}(E)=\operatorname{Per}(E)
$$

see [20], [6].

Recently, the $s$-perimeter has inspired a variety of literature in different directions (see [14], [19], [20], [24], [33], [34] and the references therein). Our aim here is to deal with non-singular kernels. As for the case of singular kernels, we will prove that the usual notion of perimeter is recovered by a limit rescaling formula:

where $J_{\epsilon}$ are the rescaled kernels

$$
\lim _{\epsilon \downarrow 0} \frac{C_{J}}{\epsilon} P_{J_{\epsilon}}(E)=\lim _{\epsilon \downarrow 0} C_{J} \epsilon^{N-1} P_{J}\left(\frac{E}{\epsilon}\right)=\operatorname{Per}(E),
$$

and the constant $C_{J}$ is given by

$$
J_{\epsilon}(x):=\frac{1}{\epsilon^{N}} J\left(\frac{x}{\epsilon}\right)
$$

$$
C_{J}=\frac{2}{\int_{\mathbb{R}^{N}} J(z)\left|z_{N}\right| d z} .
$$

Let $\Omega$ an open bounded subset of $\mathbb{R}^{N}$. We say that a measurable set $E \subset \mathbb{R}^{N}$ is $J$-minimal in $\Omega$ if

$$
P_{J}(E, \Omega) \leq P_{J}(F, \Omega) \text { for any measurable set } F \text { such that } F \backslash \Omega=E \backslash \Omega \text {. }
$$

We will see that such a set verifies the equation (Theorem 3.4)

$$
\int_{\mathbb{R}^{N}} J(x-y)\left(\chi_{\mathbb{R}^{N} \backslash E}(y)-\chi_{E}(y)\right) d y=0,
$$


for all $x \in \partial E \cap \Omega$ such that $\left|E \cap B_{\delta}(x)\right|>0$ and $\left|\left(\mathbb{R}^{N} \backslash E\right) \cap B_{\delta}(x)\right|>0$ for every small $\delta$.

So, by analogy with the classical case, we consider the left hand side of (1.4) as a nonlocal mean curvature, denoted by $H_{\partial E}^{J}(x)$ (see Section 3 for the details). For this nonlocal curvature we can also show an approximation result to recover the usual notion of mean curvature. In fact, when $E \subset \mathbb{R}^{N}$ a smooth set with $C^{2}$ boundary, for every $x \in \partial E$, we have

$$
\lim _{\epsilon \downarrow 0} \frac{C_{J}}{\epsilon} H_{\partial E}^{J_{\epsilon}}(x)=(N-1) H_{\partial E}(x),
$$

where $H_{\partial E}(x)$ is the (local) mean curvature of $\partial E$ at $x$.

Note that both the nonlocal perimeter and the nonlocal mean curvature at a point $x$ are continuous with as functions of $E$ in the following sense: if $E_{n} \rightarrow E$ in the sense that $\left|E_{n} \triangle E\right| \rightarrow 0$ then

$$
P_{J}\left(E_{n}\right) \rightarrow P_{J}(E) \quad \text { and } \quad H_{\partial E_{n}}^{J}(x) \rightarrow H_{\partial E}^{J}(x) .
$$

We also introduce the notion of nonlocal Cheeger constant (we will call it $J$-Cheeger constant) of a non-null measurable bounded $\Omega \subset \mathbb{R}^{N}$ by

$$
h_{1}^{J}(\Omega):=\inf \left\{\frac{P_{J}(E)}{|E|}: E \subset \Omega, E \text { measurable with }|E|>0\right\} .
$$

A measurable set $E_{\Omega} \subset \Omega$ achieving the infimun in (1.5) is called a $J$-Cheeger set of $\Omega$. Note that, due to the lack of compactness (that is due to the fact that here we are considering non-singular kernels) we find examples of convex sets without a $J$-Cheeger set inside. Associated with this notion of Cheeger set we have $J$-calibrable sets (a $J$-Cheeger set of itself, see Section 6 for details). Concerning examples, we show that balls are $J$-calibrable for kernels $J$ that are radially nonincresing. We give a characterization of these sets by means of a nonlocal version of the 1-Laplacian operator. We show that a set $\Omega$ is $J$-calibrable if and only if there exists $\tau$ such that $\tau(x)=1$ if $x \in \Omega$ and

$$
-\lambda_{\Omega}^{J} \tau \in \Delta_{1}^{J} \chi_{\Omega}
$$

where $\lambda_{\Omega}^{J}$ is the $J$-Cheeger constant $\lambda_{\Omega}^{J}=\frac{P_{J}(\Omega)}{|\Omega|}$ and, formally,

$$
\Delta_{1}^{J} u(x)=\int_{\mathbb{R}^{N}} J(x-y) \frac{u(y)-u(x)}{|u(y)-u(x)|} d y .
$$

Moreover, $J$-calibrable sets are related to the nonlocal curvature defined previously. In fact, when a set $\Omega$ is $J$-calibrable then

$$
H_{\partial \Omega}^{J}(x)=-\int_{\mathbb{R}^{N}} J(x-y)\left(\chi_{\Omega}(y)-\chi_{\mathbb{R}^{N} \backslash \Omega}(y)\right) d y \leq \lambda_{\Omega}^{J}=\frac{P_{J}(\Omega)}{|\Omega|},
$$

for every $x \in \Omega$.

The paper is organized as follow: In Section 2 we show some properties (such as a co-area formula and an isoperimetric inequality) of the nonlocal perimeter and provide some simple examples where it can be explicitly computed, we also prove here that we recover the usual notion of perimeter when one rescales the kernel. In Section 3 we deal with nonlocal minimal surfaces, introduce the nonlocal mean curvature and show that again under rescaling we can recover the usual notion of local mean curvature. In Section 4 we introduce some nonlocal operators that are used to give a characterization of the nonlocal perimeter. In Section 5 we study the Cauchy problem for the nonlocal 1-Laplacian. Finally, in Section 6 we analyze the nonlocal Cheeger sets and the nonlocal calibrable sets showing that again we recover the usual local results when we rescale the kernel. We also include in this last section our characterizations 
of $J$-calibrability and the proof of the fact that balls and small stadiums are $J$-calibrable while large stadiums are not.

\section{Nonlocal Perimeter}

Associated with $J$ we have introduced the nonlocal interaction beteween to sets $A$ and $B$ in (1.3) as

$$
L_{J}(A, B):=\int_{A} \int_{B} J(x-y) d y d x \text { for measurable sets } A, B \subset \mathbb{R}^{N},
$$

and a concept of perimeter of a set in that can be written as

$$
P_{J}(E)=L_{J}\left(E, \mathbb{R}^{N} \backslash E\right) .
$$

Let us now give some properties of this nonlocal perimeter. The first ones are easy to obtain.

Proposition 2.1. It holds that

$$
P_{J}(E) \leq|E| \text { for all set } E \text { of Lebesgue finite measure }
$$

and

$$
P_{J}(E)=P_{J}(z+E) \quad \text { for all } z \in \mathbb{R}^{N} \text { and all set } E \text { of Lebesgue finite measure. }
$$

Proposition 2.2. Let $A, B \subset \mathbb{R}^{N}$ be measurable sets with $A \cap B=\emptyset$. Then,

$$
P_{J}(A \cup B)=P_{J}(A)+P_{J}(B)-2 L_{J}(A, B) .
$$

In particular, if

$$
d(A ; B):=\inf \{|x-y|: x \in A, y \in B\}>\frac{1}{2} \operatorname{diam}(\operatorname{supp}(J))
$$

then

$$
P_{J}(A \cup B)=P_{J}(A)+P_{J}(B) .
$$

Proof. We have

$$
\begin{aligned}
P_{J}(A \cup B)=\int_{A \cup B}\left(\int_{\mathbb{R}^{N} \backslash(A \cup B)} J(x-y) d y\right) d x \\
=\int_{A}\left(\int_{\mathbb{R}^{N} \backslash(A \cup B)} J(x-y) d y\right) d x+\int_{B}\left(\int_{\mathbb{R}^{N} \backslash(A \cup B)} J(x-y) d y\right) d x \\
=\int_{A}\left(\int_{\mathbb{R}^{N} \backslash A} J(x-y) d y-\int_{B} J(x-y) d y\right) d x+\int_{B}\left(\int_{\mathbb{R}^{N} \backslash B} J(x-y) d y-\int_{A} J(x-y) d y\right) d x \\
=P_{J}(A)+P_{J}(B)-2 \int_{A}\left(\int_{B} J(x-y) d y\right) d x,
\end{aligned}
$$

as we wanted to show.

Corollary 2.3. Let $A, B, C$ be pairwise disjoints measurable sets in $\mathbb{R}^{N}$. Then

$$
P_{J}(A \cup B \cup C)=P_{J}(A \cup B)+P_{J}(A \cup C)+P_{J}(B \cup C)-P_{J}(A)-P_{J}(B)-P_{J}(C) .
$$

For the nonlocal perimeter there is an isoperimetric inequality when $J$ is radially decreasing. We will write $\omega_{N}$ for the Lebesgue measure of the unit ball, $\omega_{N}:=\left|B_{1}\right|$. 
Theorem 2.4 (Isoperimetric Inequality). Let $J$ be a nonnegative radially nonincreasing function. For every measurable set $E$ with finite measure it holds that

$$
\frac{P_{J}(E)}{|E|} \geq \frac{P_{J}\left(B_{r}\right)}{\left|B_{r}\right|}
$$

where $B_{r}$ is a ball such that $\left|B_{r}\right|=|E|$.

Proof. The proof uses the symmetric decreasing rearrangement, which replaces a given nonnegative function $f$ by a radial function $f^{*}$. Let us recall briefly the definition and some basic properties of this rearrangement. Let $E$ be a measurable set of finite measure. Its symmetric rearrangement $E^{*}$ is given by the open centered ball whose measure agrees with $|E|$, that is, $E^{*}=B_{r}$ if $r$ is such that $\left|B_{r}\right|=\omega_{N} r^{N}=|E|$. Now for a nonnegative and measurable function $f$ that vanishes at infinity, in the sense that all its positive level sets have finite measure, we define define the symmetric decreasing rearrangement $f^{*}$ by symmetrizing its the level sets,

$$
f^{*}(x)=\int_{0}^{\infty} \chi_{\{f(x)>t\} *} d t
$$

Note that for a radially nonincreasing function it holds that $f^{*}=f$ and that the previous definitions are consistent in the sense that

$$
\chi_{A^{*}}=\left(\chi_{A}\right)^{*} .
$$

We refer to [28] and [30] for details.

For the rearrangement of functions interacting with a convolution we have the Riesz rearrangement inequality [30, Theorem 3.7], namely,

$$
\int_{\mathbb{R}^{N}} f(x)(g * h)(x) d x \leq \int_{\mathbb{R}^{N}} f^{*}(x)\left(g^{*} * h^{*}\right)(x) d x .
$$

Now, using this inequality, for the nonlocal perimeter defined above, one has

$$
\begin{aligned}
P_{J}(E) & :=\int_{E}\left(\int_{\mathbb{R}^{N} \backslash E} J(x-y) d y\right) d x=|E|-\int_{E}\left(\int_{E} J(x-y) d y\right) d x \\
= & |E|-\int_{\mathbb{R}^{N}} \chi_{E}(x)\left(J * \chi_{E}\right)(x) d x \geq|E|-\int_{\mathbb{R}^{N}}\left(\chi_{E}\right)^{*}(x)\left(J^{*} *\left(\chi_{E}\right)^{*}\right)(x) d x \\
& =\left|B_{r}\right|-\int_{\mathbb{R}^{N}} \chi_{B_{r}}(x)\left(J * \chi_{B_{r}}\right)(x) d x=P^{J}\left(B_{r}\right),
\end{aligned}
$$

and we conclude (2.2) from the fact that $|E|=\left|B_{r}\right|$.

Proposition 2.5. For every $u \in B V\left(\mathbb{R}^{N}\right)$ we have

$$
\mathcal{F}_{J}(u) \leq \frac{1}{2} \mathcal{F}(u)
$$

In particular, for every set of finite perimeter $E \subset \mathbb{R}^{N}$,

$$
P_{J}(E) \leq \frac{1}{2} \operatorname{Per}(E) .
$$

Proof. Given $u \in B V\left(\mathbb{R}^{N}\right)$ there exists a sequence $\left\{u_{n}\right\}_{n \in \mathbb{N}} \subset C^{\infty}\left(\mathbb{R}^{N}\right) \cap B V\left(\mathbb{R}^{N}\right)$ such that

$$
\lim _{n \rightarrow \infty}\left\|u_{n}-u\right\|_{L^{1}\left(\mathbb{R}^{N}\right)}=0 \quad \lim _{n \rightarrow \infty} \int_{\mathbb{R}^{N}}\left|\nabla u_{n}\right| d x=\int_{\mathbb{R}^{N}}|D u| .
$$


Then, in order to prove (2.3) it will be sufficient to prove it for $u \in C^{\infty}\left(\mathbb{R}^{N}\right) \cap B V\left(\mathbb{R}^{N}\right)$.

$$
\mathcal{F}_{J}(u)=\frac{1}{2} \int_{B_{1}(0)} J(z) \int_{\mathbb{R}^{N}}|u(x+z)-u(x)| d x d z .
$$

Observe that we have

$$
|u(x+z)-u(x)| \leq\left(\int_{0}^{1}|\nabla u(x+t z)| d t\right)|z| .
$$

Hence

$$
\begin{aligned}
\mathcal{F}_{J}(u) & \leq \frac{1}{2} \int_{B_{1}(0)} J(z) \int_{\mathbb{R}^{N}}\left(\int_{0}^{1}|\nabla u(x+t z)| d t\right)|z| d x d z \\
\leq & \frac{1}{2} \int_{B_{1}(0)} J(z) \int_{0}^{1}\left(\int_{\mathbb{R}^{N}}|\nabla u(x+t z)| d x\right) d t d z \\
\leq & \frac{1}{2} \int_{B_{1}(0)} J(z)\left(\int_{\mathbb{R}^{N}}|\nabla u(\xi)| d \xi\right) d z=\frac{1}{2} \int_{\mathbb{R}^{N}}|\nabla u(\xi)| d \xi .
\end{aligned}
$$

Theorem 2.6 (Coarea formula). For any $u \in L^{1}\left(\mathbb{R}^{N}\right)$, let $E_{t}(u):=\left\{x \in \mathbb{R}^{N}: u(x)>t\right\}$. Then

$$
\mathcal{F}_{J}(u)=\int_{-\infty}^{+\infty} P_{J}\left(E_{t}(u)\right) d t
$$

Proof. Since

$$
u(x)=\int_{0}^{\infty} \chi_{E_{t}(u)}(x) d t-\int_{-\infty}^{0}\left(1-\chi_{E_{t}(u)}(x)\right) d t
$$

we have

$$
u(y)-u(x)=\int_{-\infty}^{+\infty} \chi_{E_{t}(u)}(y)-\chi_{E_{t}(u)}(x) d t .
$$

Moreover, since $u(y) \geq u(x)$ imply $\chi_{E_{t}(u)}(y) \geq \chi_{E_{t}(u)}(x)$, we obtain that

$$
|u(y)-u(x)|=\int_{-\infty}^{+\infty}\left|\chi_{E_{t}(u)}(y)-\chi_{E_{t}(u)}(x)\right| d t .
$$

Therefore, by Tonelli-Hobson's Theorem, we get

$$
\begin{aligned}
\mathcal{F}_{J}(u) & =\frac{1}{2} \int_{\mathbb{R}^{N}} \int_{\mathbb{R}^{N}} J(x-y)|u(y)-u(x)| d x d y \\
= & \frac{1}{2} \int_{\mathbb{R}^{N}} \int_{\mathbb{R}^{N}} J(x-y)\left(\int_{-\infty}^{+\infty}\left|\chi_{E_{t}(u)}(y)-\chi_{E_{t}(u)}(x)\right| d t\right) d x d y \\
= & \int_{-\infty}^{+\infty}\left(\frac{1}{2} \int_{\mathbb{R}^{N}} \int_{\mathbb{R}^{N}} J(x-y)\left|\chi_{E_{t}(u)}(y)-\chi_{E_{t}(u)}(x)\right| d x d y\right) d t=\int_{-\infty}^{+\infty} P_{J}\left(E_{t}(u)\right) d t .
\end{aligned}
$$

We now consider the rescaled kernel

$$
J_{\epsilon}(x):=\frac{1}{\epsilon^{N}} J\left(\frac{x}{\epsilon}\right) .
$$


Observe that if $\operatorname{supp}(J)=B_{r}(0)$ then $J_{\epsilon}$ is supported in $B_{r \epsilon}(0)$, has the same total mass as $J$, and verifies

$$
P_{J}\left(\frac{1}{\epsilon} E\right)=\frac{1}{\epsilon^{N}} P_{J_{\epsilon}}(E) \quad \text { for all set } E \text { of Lebesgue finite measure. }
$$

Let also

$$
C_{J}=\frac{2}{\int_{\mathbb{R}^{N}} J(z)\left|z_{N}\right| d z}
$$

In [11] it is proved that the solutions $u_{\epsilon}$ of equations of the form

$$
u_{t}(t, x)=\frac{C_{J}}{\epsilon} \int J_{\epsilon}(x-y) \frac{u(t, y)-u(t, x)}{|u(t, y)-u(t, x)|} d y
$$

converge (up to a subsequence) to the solution of

$$
u_{t}=\Delta_{1} u
$$

for different boundary conditions, being $\Delta_{1} u:=\operatorname{div}\left(\frac{D u}{|D u|}\right)$. Our aim is to study which is the behaviour of the nonlocal $J$-perimeter under rescaling.

Example 2.7. Take $J:=\frac{1}{2} \chi_{[-1,1]}$. Then, a simple calculation gives

$$
P_{J}([a, b])=\frac{1}{2}\left(1-\left((1-(b-a))^{+}\right)^{2}\right)= \begin{cases}\frac{1}{2} & \text { if } b-a>1 \\ (b-a)\left(1-\frac{1}{2}(b-a)\right) & \text { if } b-a \leq 1 .\end{cases}
$$

Let us compute $\lim _{\epsilon \rightarrow 0} \frac{C_{J}}{\epsilon} P_{J_{\epsilon}}(E)$. Observe that for this particular kernel we have $C_{J}=4$. On account of (2.5) and (2.6), for $\epsilon$ small, we have

$$
\frac{4}{\epsilon} P_{J_{\epsilon}}([a, b])=4 P_{J}\left(\left[\frac{a}{\epsilon}, \frac{b}{\epsilon}\right]\right)=4 \times \frac{1}{2}=2=\operatorname{Per}([a, b]) .
$$

Example 2.8. Let now $J(x):=w(\|x\|) \chi_{B_{R}(0)}(x)$. If $w(r)=C_{R} r^{\alpha}$ then

$$
\begin{gathered}
\int_{\mathbb{R}^{N}} J(x) d x=C_{R} \int_{B_{R}(0)}\|x\|^{\alpha} d x=C_{R} \int_{0}^{R}\left(\int_{\partial B_{r}(0)} r^{\alpha} d \sigma\right) d r=C_{R} N \omega_{N} \int_{0}^{R} r^{\alpha+N-1} d r \\
=\frac{C_{R} N \omega_{N}}{N+\alpha} R^{N+\alpha}=C_{R} \frac{N\left|B_{R}(0)\right| R^{\alpha}}{N+\alpha} .
\end{gathered}
$$

Then, if $\alpha>-N$ and $C_{R}=\frac{N+\alpha}{N\left|B_{R}(0)\right| R^{\alpha}}$, we have $\int_{\mathbb{R}^{N}} J(x) d x=1$. Consider the case $N=1, R=1$ and $\alpha=-\frac{1}{2}$, which corresponds to

$$
J(x)=\frac{1}{4 \sqrt{|x|}} \chi_{[-1,1]}(x)
$$

Then, a simple calculation gives

$$
P_{J}([a, b])= \begin{cases}b-a-\frac{2}{3}(b-a)^{\frac{3}{2}} & \text { if } b-a<1 \\ \frac{1}{3} & \text { if } b-a \geq 1 .\end{cases}
$$


Now $C_{J}=6$ and, on account of (2.5) and (2.7), for $\epsilon$ small, we have

$$
\frac{C_{J}}{\epsilon} P_{J_{\epsilon}}([a, b])=\frac{6}{\epsilon} P_{J_{\epsilon}}([a, b])=6 P_{J}\left(\left[\frac{a}{\epsilon}, \frac{b}{\epsilon}\right]\right)=6 \cdot \frac{1}{3}=2=\operatorname{Per}([a, b]) .
$$

Therefore, in these two above examples we have obtained that

$$
\lim _{\epsilon \downarrow 0} \frac{C_{J}}{\epsilon} P_{J_{\epsilon}}(E)=\operatorname{Per}(E) .
$$

We will now see that (2.8) is always true as a consequence of the following result by J. Dávila (see also [17] and [31]).

Theorem 2.9 (Dávila [21]). Let $B \subset \mathbb{R}^{N}$ be open, bounded with a Lipschitz boundary, and let $0 \leq \rho_{\epsilon}$ radial functions satisfying

$$
\int_{\mathbb{R}^{N}} \rho_{\epsilon}(x) d x=1, \quad \lim _{\epsilon \rightarrow 0} \int_{\delta}^{\infty} \tilde{\rho}_{\epsilon}(r) r^{N-1} d r=0, \quad \forall \delta>0,
$$

where $\tilde{\rho}_{\epsilon}(r)=\rho_{\epsilon}(x)$ for $|x|=r$. Then

$$
\lim _{\epsilon \rightarrow 0} \int_{B} \int_{B} \frac{|u(x)-u(y)|}{|x-y|} \rho_{\epsilon}(x-y) d x d y=K_{1, N} \int_{B}|D u|,
$$

where

$$
K_{1, N}=\frac{1}{N \omega_{N}} \int_{S^{N-1}}|e \cdot \sigma| d \sigma=\frac{\Gamma\left(\frac{N}{2}\right)}{\sqrt{\pi} \Gamma\left(\frac{N+1}{2}\right)}, \quad|e|=1 .
$$

With this result at hand we can prove the following convergence result as we rescale the kernel $J$.

Theorem 2.10. Let $J$ be compactly supported. If $u \in B V\left(\mathbb{R}^{N}\right)$ has compact support then

$$
\lim _{\epsilon \downarrow 0} \frac{C_{J}}{\epsilon} \mathcal{F}_{J_{\epsilon}}(u)=\int_{\mathbb{R}^{N}}|D u| .
$$

In particular, if $E \subset \mathbb{R}^{N}$ is a bounded set of finite perimeter then

$$
\lim _{\epsilon \downarrow 0} \frac{C_{J}}{\epsilon} P_{J_{\epsilon}}(E)=\lim _{\epsilon \downarrow 0} C_{J} \epsilon^{N-1} P_{J}\left(\frac{E}{\epsilon}\right)=\operatorname{Per}(E) .
$$

Proof. Since $u$ has compact support, for a large ball $B$ containing $\operatorname{supp}(u)$ we can rewrite

$$
\mathcal{F}_{J_{\epsilon}}(u)=\frac{1}{2} \int_{B} \int_{B} J_{\epsilon}(x-y)|u(y)-u(x)| d y d x \quad \text { and } \quad \int_{\mathbb{R}^{N}}|D u|=\int_{B}|D u| .
$$

Now,

$$
\frac{C_{J}}{\epsilon} \mathcal{F}_{J_{\epsilon}}(u)=\frac{1}{K_{1, N}} \int_{B} \int_{B} \frac{|u(x)-u(y)|}{|x-y|} \rho_{\epsilon}(x-y) d x d y,
$$

being

$$
\rho_{\epsilon}(z)=\frac{1}{2} C_{J} K_{1, N} \frac{|z|}{\epsilon} J_{\epsilon}(z)
$$


Then, denoting $\tilde{J}(r)=J(x)$ if $|x|=r$,

$$
\begin{aligned}
\int_{\mathbb{R}^{N}} \rho_{\epsilon}(z) d z=\frac{1}{2} C_{J} K_{1, N} \int_{\mathbb{R}^{N}} \frac{|z|}{\epsilon} \frac{1}{\epsilon^{N}} J\left(\frac{z}{\epsilon}\right) d z \\
=K_{1, N} \frac{\int_{\mathbb{R}^{N}}|w| J(w) d w}{\int_{\mathbb{R}^{N}}\left|w_{N}\right| J(w) d w} \\
=K_{1, N} \frac{\int_{0}^{\infty} \int_{\partial B_{r}(0)} r \tilde{J}(r) d \sigma d r}{\int_{0}^{\infty} \int_{\partial B_{r}(0)} \tilde{J}(r)\left|\sigma \cdot e_{N}\right| d \sigma d r} \\
=\frac{\int_{S^{N-1}}\left|e_{N} \cdot \sigma\right| d \sigma}{N \omega_{N}} \cdot \frac{N \omega_{N} \int_{0}^{\infty} r^{N} \tilde{J}(r) d r}{\int_{0}^{\infty} \tilde{J}(r) r^{N} \int_{S^{N-1}}\left|e_{N} \cdot \sigma\right| d \sigma d r}=1
\end{aligned}
$$

and, for $\delta>0$,

$$
\lim _{\epsilon \rightarrow 0} \int_{\delta}^{\infty} \tilde{\rho}_{\epsilon}(r) r^{N-1} d r=\lim _{\epsilon \rightarrow 0} \int_{\frac{\delta}{\epsilon}}^{\infty} r^{N} \tilde{J}(r) d r=0
$$

since $J$ has compact support. Therefore, we can apply Theorem 2.9 to get the result.

\section{Nonlocal minimal surfaces}

Let $\Omega$ an open bounded subset of $\mathbb{R}^{N}$. We say that a measurable set $E \subset \mathbb{R}^{N}$ is $J$-minimal in $\Omega$ if

$$
P_{J}(E, \Omega) \leq P_{J}(F, \Omega) \text { for any measurable set } F \text { such that } F \backslash \Omega=E \backslash \Omega \text {. }
$$

Proposition 3.1. Let $\Omega$ an open bounded subset of $\mathbb{R}^{N}$. Then, $E \subset \mathbb{R}^{N}$ is $J$-minimal in $\Omega$ if and only if

$$
P_{J}(E) \leq P_{J}(F) \text { for any measurable set } F \text { such that } F \backslash \Omega=E \backslash \Omega \text {. }
$$

Proof. If $E \backslash \Omega=F \backslash \Omega$ then $\mathbb{R}^{N} \backslash(\Omega \cup E)=\mathbb{R}^{N} \backslash(\Omega \cup F)$. Therefore, since

$$
P_{J}(A, \Omega)=L_{J}\left(A, \mathbb{R}^{N} \backslash A\right)-L_{J}\left(A \backslash \Omega, \mathbb{R}^{N} \backslash(A \cup \Omega)\right)
$$

we get the result.

This is a nonlocal version of a set with minimal perimeter. Recall that if a set $E$ has minimal (local) perimeter in a bounded set $\Omega$, then it has zero mean curvature at each point of $\partial E \cap \Omega$ (see [27]), and the curvature is the Euler-Lagrange equation associated to the minimization of the perimeter of a set. In order to give a nonlocal version of this result we introduce the following nonlocal concept of mean curvature.

Definition 3.2. Assume $N \geq 2$ and let $E \subset \mathbb{R}^{N}$ be measurable. For a point $x \in \mathbb{R}^{N}$ we define its $J$-mean curvature as

$$
H_{\partial E}^{J}(x):=-\int_{\mathbb{R}^{N}} J(x-y)\left(\chi_{E}(y)-\chi_{\mathbb{R}^{N} \backslash E}(y)\right) d y .
$$

Note that $H_{\partial E}^{J}(x)$ makes perfect sense for every $x \in \mathbb{R}^{N}$, not necessary for points in $\partial E$. This fact will be used later in the paper.

Like the usual mean curvature, if $\partial E$ is a smooth boundary, for $x \in \partial E, H_{\partial E}^{J}(x)$ measures in some average sense the deviation of $\partial E$ from its tangent hyperplane at $x$. Let us point out that with our choice, 
the curvature of a ball is positive as is common in geometry texts. Some authors define the curvature with the reverse sign, see for example, [1] and [18].

Definition 3.3. We say that $\partial E$ is a J-minimal surface in a bounded open set $\Omega$ if the set $\partial E \cap \Omega$ satisfies the nonlocal minimal surface equation

$$
H_{\partial E}^{J}(x)=0 \quad \forall x \in \partial E \cap \Omega:\left|E \cap B_{\delta}(x)\right|>0 \text { and }\left|\left(\mathbb{R}^{N} \backslash E\right) \cap B_{\delta}(x)\right|>0 \text { for every small } \delta .
$$

As examples of nonlocal minimal surfaces we have the following: By symmetry and (3.1) any hyperplane, $E=\left\{x \in \mathbb{R}^{N}: x_{N}>0\right\}$, is a $J$-minimal surface in $\mathbb{R}^{N}$. Similarly, the classical cone in the plane, $E:=\left\{(x, y) \in \mathbb{R}^{2}: x y>0\right\}$, is a $J$-minimal surface in $\mathbb{R}^{2}$.

Theorem 3.4. Let $\Omega$ an open bounded subset of $\mathbb{R}^{N}$. If $E \subset \mathbb{R}^{N}$ a J-minimal set in $\Omega$ then $\partial E$ is a $J$-minimal surface in $\Omega$.

Proof. Let $E \subset \mathbb{R}^{N}$ a $J$-minimal set in $\Omega$. Given $x_{0} \in \partial E \cap \Omega$, there is $\delta>0$ such that $B_{\delta}\left(x_{0}\right) \subset \Omega$. Now consider

$$
A_{\delta}=B_{\delta}\left(x_{0}\right) \cap\left(\mathbb{R}^{N} \backslash E\right)
$$

and

$$
F_{\delta}=A_{\delta} \cup E .
$$

Since, $F_{\delta} \backslash \Omega=E \backslash \Omega$, we have

$$
P_{J}(E) \leq P_{J}\left(F_{\delta}\right)
$$

that is

$$
\int_{E} \int_{\mathbb{R}^{N} \backslash E} J(x-y) d y d x \leq \int_{F_{\delta}} \int_{\mathbb{R}^{N} \backslash F_{\delta}} J(x-y) d y d x .
$$

Now, since $A_{\delta} \cap E=\emptyset$,

$$
\int_{E} \int_{\mathbb{R}^{N} \backslash E} J(x-y) d y d x=\int_{F_{\delta}} \int_{\mathbb{R}^{N} \backslash E} J(x-y) d y d x-\int_{A_{\delta}} \int_{\mathbb{R}^{N} \backslash E} J(x-y) d y d x .
$$

Therefore

$$
\begin{aligned}
\int_{A_{\delta}} & \int_{\mathbb{R}^{N} \backslash E} J(x-y) d y d x \geq \int_{F_{\delta}}\left(\int_{\mathbb{R}^{N} \backslash E} J(x-y) d y-\int_{\mathbb{R}^{N} \backslash F_{\delta}} J(x-y) d y\right) d x \\
& =\int_{F_{\delta}}\left(\int_{A_{\delta}} J(x-y) d y\right) d x=\int_{A_{\delta}} \int_{A_{\delta}} J(x-y) d y d x+\int_{E} \int_{A_{\delta}} J(x-y) d y d x .
\end{aligned}
$$

Hence

$$
\int_{A_{\delta}} \int_{A_{\delta}} J(x-y) d y d x \leq \int_{A_{\delta}}\left(\int_{\mathbb{R}^{N} \backslash E} J(x-y) d y-\int_{E} J(x-y) d y\right) d x .
$$

Now, since $x \in \partial E$, we have $\left|A_{\delta}\right|>0$. Then, dividing by $\left|A_{\delta}\right|$ and letting $\delta \rightarrow 0$, we conclude that

$$
\left(\int_{\mathbb{R}^{N} \backslash E} J\left(x_{0}-y\right) d y-\int_{E} J\left(x_{0}-y\right) d y\right) \geq 0 .
$$

With a similar procedure, but taking now $\tilde{A}_{\delta}:=B_{\delta}\left(x_{0}\right) \cap E$ and $\tilde{F}_{\delta}:=E \backslash \tilde{A}_{\delta}$, we arrive to

$$
\int_{\tilde{A}_{\delta}}\left(\int_{\mathbb{R}^{N} \backslash E} J(x-y) d y-\int_{E} J(x-y) d y\right) d x \leq-\int_{\tilde{A}_{\delta}} \int_{\tilde{A}_{\delta}} J(x-y) d y d x .
$$


Then, dividing by $\left|\tilde{A}_{\delta}\right|>0$ and letting $\delta \rightarrow 0$, we get

$$
\left(\int_{\mathbb{R}^{N} \backslash E} J\left(x_{0}-y\right) d y-\int_{E} J\left(x_{0}-y\right) d y\right) \leq 0 .
$$

Therefore, as we wanted to show,

$$
H_{\partial E}^{J}\left(x_{0}\right)=-\int_{\mathbb{R}^{N}} J\left(x_{0}-y\right)\left(\chi_{E}(y)-\chi_{\mathbb{R}^{N} \backslash E}(y)\right) d y=0 .
$$

Remark 3.5. Let us point out that for the singular case, a similar result to Theorem 3.4 has been obtained in [1] and [18] for singular kernels, where the nonlocal curvature at $x \in \partial E$ is defined as

$$
\int_{\mathbb{R}^{N}} \frac{\chi_{E}(y)-\chi_{\mathbb{R}^{N} \backslash E}(y)}{|x-y|^{N+s}} d y .
$$

Our definition of curvature differs from the above one, besides of the kernel, in the sign, but makes the curvature of the ball positive.

Example 3.6. If $J(x)=\frac{1}{\left|B_{1}(0)\right|} \chi_{B_{1}(0)}$, then for any $x \in \overline{B_{r}\left(x_{0}\right)}$, we have

$$
\begin{aligned}
& H_{\partial B_{r}\left(x_{0}\right)}^{J}(x)=-\int_{\mathbb{R}^{N}} J(x-y)\left(\chi_{B_{r}\left(x_{0}\right)}(y)-\chi_{\mathbb{R}^{N} \backslash B_{r}\left(x_{0}\right)}(y)\right) d y \\
& =-\frac{1}{\left|B_{1}(0)\right|} \int_{B_{1}(x)}\left(\chi_{B_{r}\left(x_{0}\right)}(y)-\chi_{\mathbb{R}^{N} \backslash B_{r}\left(x_{0}\right)}(y)\right) d y \\
& =-\frac{1}{\left|B_{1}(0)\right|}\left(\left|B_{1}(x) \cap B_{r}\left(x_{0}\right)\right|-\left|B_{1}(x) \cap\left(\mathbb{R}^{N} \backslash B_{r}\left(x_{0}\right)\right)\right|\right) \\
& =-\frac{1}{\left|B_{1}(0)\right|}\left(2\left|B_{1}(x) \cap B_{r}\left(x_{0}\right)\right|-\left|B_{1}(x)\right|\right) \\
& =-\frac{2\left|B_{1}(x) \cap B_{r}\left(x_{0}\right)\right|}{\left|B_{1}(0)\right|}+1 .
\end{aligned}
$$

And for the rescaled kernel,

$$
\begin{aligned}
& H_{\partial B_{r}\left(x_{0}\right)}^{J_{\epsilon}}(x)=-\frac{1}{\epsilon^{N}} \int_{\mathbb{R}^{N}} J\left(\frac{x-y}{\epsilon}\right)\left(\chi_{B_{r}\left(x_{0}\right)}(y)-\chi_{\mathbb{R}^{N} \backslash B_{r}\left(x_{0}\right)}(y)\right) d y \\
& \quad=-\frac{1}{\left|B_{\epsilon}(0)\right|} \int_{B_{\epsilon}(x)}\left(\chi_{B_{r}\left(x_{0}\right)}(y)-\chi_{\mathbb{R}^{N} \backslash B_{r}\left(x_{0}\right)}(y)\right) d y \\
& \quad=-\frac{1}{\left|B_{\epsilon}(0)\right|}\left(\left|B_{\epsilon}(x) \cap B_{r}\left(x_{0}\right)\right|-\left|B_{\epsilon}(x) \cap\left(\mathbb{R}^{N} \backslash B_{r}\left(x_{0}\right)\right)\right|\right) \\
& =-\frac{1}{\left|B_{\epsilon}(0)\right|}\left(2\left|B_{\epsilon}(x) \cap B_{r}\left(x_{0}\right)\right|-\left|B_{\epsilon}(x)\right|\right) \\
& =-\frac{2\left|B_{\epsilon}(x) \cap B_{r}\left(x_{0}\right)\right|}{\left|B_{\epsilon}(0)\right|}+1 .
\end{aligned}
$$


Now, for $N=2$, a simple calculus gives, for $x \in \partial B_{r}\left(x_{0}\right)$ and $\epsilon$ small,

$$
\begin{aligned}
& \left|B_{\epsilon}(x) \cap B_{r}\left(x_{0}\right)\right|=\epsilon^{2}\left[-\frac{\epsilon}{2 r} \sqrt{1-\left(\frac{\epsilon}{2 r}\right)^{2}}+\arcsin \left(-\frac{\epsilon}{2 r}\right)+\frac{\pi}{2}\right] \\
& \quad+r^{2}\left[\frac{\pi}{2}-\frac{2 r^{2}-\epsilon^{2}}{2 r^{2}} \sqrt{1-\left(\frac{2 r^{2}-\epsilon^{2}}{2 r^{2}}\right)^{2}}-\arcsin \left(\frac{2 r^{2}-\epsilon^{2}}{2 r^{2}}\right)\right] .
\end{aligned}
$$

Hence

$$
\begin{aligned}
& H_{\partial B_{r}\left(x_{0}\right)}^{J_{\epsilon}}(x)=\frac{\epsilon}{\pi r} \sqrt{1-\left(\frac{\epsilon}{2 r}\right)^{2}}+\frac{2}{\pi} \arcsin \left(\frac{\epsilon}{2 r}\right) \\
& -\frac{2 r^{2}}{\pi \epsilon^{2}}\left[\frac{\pi}{2}-\frac{2 r^{2}-\epsilon^{2}}{2 r^{2}} \sqrt{1-\left(\frac{2 r^{2}-\epsilon^{2}}{2 r^{2}}\right)^{2}}-\arcsin \left(\frac{2 r^{2}-\epsilon^{2}}{2 r^{2}}\right)\right] .
\end{aligned}
$$

Now,

$$
C_{J}=\frac{2}{\int_{\mathbb{R}^{2}} J(z)\left|z_{2}\right| d z}=\frac{2}{\frac{1}{\left|B_{1}(0)\right|} \int_{B_{1}(0)}\left|z_{2}\right| d z}=\frac{3 \pi}{2} .
$$

Therefore,

$$
\begin{aligned}
& \frac{C_{J}}{\epsilon} H_{\partial B_{r}\left(x_{0}\right)}^{J_{\epsilon}}(x)=\frac{3}{2 r} \sqrt{1-\left(\frac{\epsilon}{2 r}\right)^{2}}+\frac{3}{\epsilon} \arcsin \left(\frac{\epsilon}{2 r}\right) \\
& \quad-\frac{3 r^{2}}{\epsilon^{3}}\left[\frac{\pi}{2}-\frac{2 r^{2}-\epsilon^{2}}{2 r^{2}} \sqrt{1-\left(\frac{2 r^{2}-\epsilon^{2}}{2 r^{2}}\right)^{2}}-\arcsin \left(\frac{2 r^{2}-\epsilon^{2}}{2 r^{2}}\right)\right] .
\end{aligned}
$$

Then, since

$$
\lim _{\epsilon \rightarrow 0} \frac{3}{\epsilon} \arcsin \left(\frac{\epsilon}{2 r}\right)=\frac{3}{2 r}
$$

and

$$
\lim _{\epsilon \rightarrow 0} \frac{3 r^{2}}{\epsilon^{3}}\left[\frac{\pi}{2}-\frac{2 r^{2}-\epsilon^{2}}{2 r^{2}} \sqrt{1-\left(\frac{2 r^{2}-\epsilon^{2}}{2 r^{2}}\right)^{2}}-\arcsin \left(\frac{2 r^{2}-\epsilon^{2}}{2 r^{2}}\right)\right]=\frac{2}{r},
$$

we get

$$
\lim _{\epsilon \rightarrow 0} \frac{C_{J}}{\epsilon} H_{\partial B_{r}\left(x_{0}\right)}^{J_{\epsilon}}(x)=\frac{3}{2 r}+\frac{3}{2 r}-\frac{2}{r}=\frac{1}{r}=H_{\partial B_{r}\left(x_{0}\right)}
$$

Consider now $E$ as the square $E:=\left\{(x, y) \in \mathbb{R}^{2}:\|(x, y)\|_{\infty} \leq 1\right\}$. Then, for $0<\epsilon<1$ we have

$$
\frac{C_{J}}{\epsilon} H_{\partial E}^{J_{\epsilon}}(1,1)=-\frac{3}{2 \epsilon^{3}}\left(\left|E \cap B_{\epsilon}(1,1)\right|-\mid\left(\mathbb{R}^{2} \backslash E \cap B_{\epsilon}(1,1) \mid\right)=\frac{3 \pi}{4 \epsilon} .\right.
$$

Therefore,

$$
\lim _{\epsilon \rightarrow 0} \frac{C_{J}}{\epsilon} H_{\partial E}^{J_{\epsilon}}(1,1)=+\infty .
$$

Theorem 3.7. Let $E \subset \mathbb{R}^{N}$ a smooth set such that $\partial E$ is of class $C^{2}$. Then, for every $x \in \partial E$, we have

$$
\lim _{\epsilon \downarrow 0} \frac{C_{J}}{\epsilon} H_{\partial E}^{J_{\epsilon}}(x)=(N-1) H_{\partial E}(x),
$$

where $H_{\partial E}(x)$ is the (local) mean curvature of $\partial E$ at $x$. 
Proof. We can assume that $x=0 \in \partial E$. It is well-known that curvature may be easily computed in normal coordinates. Namely, suppose $\partial E$ is described as a graph in normal coordinates, meaning that, in an open ball $B_{r_{0}}, \partial E$ coincides with the graph of a $C^{2}$ function $\varphi: B_{r_{0}} \cap \mathbb{R}^{N-1} \rightarrow \mathbb{R}$ with $\varphi(0)=0$ and $\nabla \varphi(0)=0$ such that $E \cap B_{r_{0}}=\left\{\left(y_{1}, \ldots, y_{N}\right): y_{N}<\varphi\left(y_{1}, \ldots, y_{N-1}\right)\right\}$. Since $D^{2} \varphi(0)$ is a real symmetric matrix, it will admit $N-1$ real eigenvalues $\lambda_{1}, \ldots, \lambda_{N-1}$. Minus the arithmetic mean of the eigenvalues is called the mean curvature and we denote it by $H_{\partial E}(0)$, namely

$$
H_{\partial E}(0):=-\frac{\lambda_{1}+\cdots+\lambda_{N-1}}{N-1}
$$

We can assume that $r=1$ and $r_{0}<1$, then

$$
\begin{gathered}
\frac{C_{J}}{\epsilon} H_{\partial E}^{J_{\epsilon}}(0):=-\frac{C_{J}}{\epsilon} \int_{\mathbb{R}^{N}} J_{\epsilon}(y)\left(\chi_{E}(y)-\chi_{\mathbb{R}^{N} \backslash E}(y)\right) d y=-\frac{C_{J}}{\epsilon^{N+1}} \int_{B_{\epsilon}(0)} J\left(\frac{y}{\epsilon}\right)\left(\chi_{E}(y)-\chi_{\mathbb{R}^{N} \backslash E}(y)\right) d y \\
=-\frac{C_{J}}{\epsilon^{N+1}}\left(\int_{\left\{y_{N}<\varphi\left(y_{1}, \ldots, y_{N-1}\right)\right\} \cap B_{\epsilon}(0)} J\left(\frac{y}{\epsilon}\right) d y-\int_{\left\{y_{N}>\varphi\left(y_{1}, \ldots, y_{N-1}\right)\right\} \cap B_{\epsilon}(0)} J\left(\frac{y}{\epsilon}\right) d y\right) .
\end{gathered}
$$

Hence, changing variables as $z=\frac{y}{\epsilon}$, we get

$$
\frac{C_{J}}{\epsilon} H_{\partial E}^{J_{\epsilon}}(0)=-\frac{C_{J}}{\epsilon}\left(\int_{\left\{z_{N}<\frac{1}{\epsilon} \varphi\left(\epsilon z_{1}, \ldots, \epsilon z_{N-1}\right)\right\} \cap B_{1}(0)} J(z) d z-\int_{\left\{z_{N}>\frac{1}{\epsilon} \varphi\left(\epsilon z_{1}, \ldots, \epsilon z_{N-1}\right)\right\} \cap B_{1}(0)} J(z) d z\right) .
$$

Now, by Taylor's expansion, we have

$$
\varphi\left(\epsilon z_{1}, \ldots, \epsilon z_{N-1}\right)=\frac{1}{2} D^{2} \varphi(0)\left(\epsilon z_{1}, \ldots, \epsilon z_{N-1}\right)=\frac{1}{2} \sum_{i=1}^{N-1} \lambda_{i} \epsilon^{2} z_{i}^{2}+O\left(\epsilon^{3}\right) .
$$

Therefore,

$$
\begin{aligned}
& \lim _{\epsilon \rightarrow 0} \frac{C_{J}}{\epsilon} H_{\partial E}^{J_{\epsilon}}(0)=-\lim _{\epsilon \rightarrow 0} \frac{C_{J}}{\epsilon}\left(\int_{\left\{z_{N}<\epsilon \frac{1}{2} \sum_{i=1}^{N-1} \lambda_{i} z_{i}^{2}\right\} \cap B_{1}(0)} J(z) d z-\int_{\left\{z_{N}>\epsilon \frac{1}{2} \sum_{i=1}^{N-1} \lambda_{i} z_{i}^{2}\right\} \cap B_{1}(0)} J(z) d z\right) \\
& =-\lim _{\epsilon \rightarrow 0} \frac{C_{J}}{\epsilon}\left(\int_{\mathbb{R}^{N-1} \cap B_{1}(0)} \int_{\left.-\sqrt{1-\left(z_{1}^{2}+\cdots+z_{N-1}^{2}\right.}\right)}^{\epsilon_{\frac{1}{2} \sum_{i=1}^{N-1} \lambda_{i} z_{i}^{2}}} J(z) d z-\int_{\mathbb{R}^{N-1} \cap B_{1}(0)} \int_{\epsilon \frac{1}{2} \sum_{i=1}^{N-1} \lambda_{i} z_{i}^{2}} J(z) d z\right) .
\end{aligned}
$$

Since the term between brackets goes to zero as $\epsilon \rightarrow 0$ we can use L'Hopital's rule to obtain

$$
\begin{aligned}
\lim _{\epsilon \rightarrow 0} & \frac{C_{J}}{\epsilon} H_{\partial E}^{J_{\epsilon}}(0)=-\lim _{\epsilon \rightarrow 0} C_{J}\left(\int_{\mathbb{R}^{N-1} \cap B_{1}(0)} J\left(z_{1}, \ldots, z_{N-1}, \epsilon \frac{1}{2} \sum_{i=1}^{N-1} \lambda_{i} z_{i}^{2}\right) \sum_{i=1}^{N-1} \lambda_{i} z_{i}^{2} d z_{1} \ldots d z_{N-1}\right) . \\
& =-C_{J} \sum_{i=1}^{N-1} \lambda_{i} \int_{\mathbb{R}^{N-1} \cap B_{1}(0)} J\left(z_{1}, \ldots, z_{N-1}, 0\right) z_{i}^{2} d z_{1} \ldots d z_{N-1} \\
& =-C_{J} \sum_{i=1}^{N-1} \lambda_{i} \int_{\mathbb{R}^{N-1} \cap B_{1}(0)} J\left(z_{1}, \ldots, z_{N-1}, 0\right) z_{N-1}^{2} d z_{1} \ldots d z_{N-1}
\end{aligned}
$$

where in the last equality we have used that $J$ is a radial function. 
On the other hand, if we made the change of variables $z_{i}=z_{i}, i=1, \ldots, N-2, z_{N-1}=r \cos \theta$, $z_{N}=r \sin \theta$, we have

$$
\begin{array}{rl}
\int_{\mathbb{R}^{N}} & J(z)\left|z_{N}\right| d z=\int_{|z| \leq 1} J(z)\left|z_{N}\right| d z \\
& =\int_{z_{1}^{2}+\cdots+z_{N-2}^{2} \leq 1} \int_{0}^{\sqrt{1-\left(z_{1}^{2}+\cdots+z_{N-2}^{2}\right)}} \int_{0}^{2 \pi} J\left(z_{1}, \ldots, z_{N-2}, r \cos \theta, r \sin \theta\right) r^{2}|\sin \theta| d z_{1} \ldots d z_{N-2} d r d \theta \\
& =4 \int_{z_{1}^{2}+\cdots+z_{N-2}^{2} \leq 1} \int_{0}^{\sqrt{1-\left(z_{1}^{2}+\cdots+z_{N-2}^{2}\right)}} J\left(z_{1}, \ldots, z_{N-2}, r, 0\right) r^{2} d z_{1} \ldots d z_{N-2} d r \\
& =2 \int_{z_{1}^{2}+\cdots+z_{N-2}^{2}+r^{2} \leq 1} J\left(z_{1}, \ldots, z_{N-2}, r, 0\right) r^{2} d z_{1} \ldots d z_{N-2} d r .
\end{array}
$$

Hence

$$
C_{J}=\frac{2}{\int_{\mathbb{R}^{N}} J(z)\left|z_{N}\right| d z}=\frac{1}{\int_{\mathbb{R}^{N-1} \cap B_{1}(0)} J\left(z_{1}, \ldots, z_{N-1}, 0\right) z_{N-1}^{2} d z_{1} \ldots d z_{N-1}} .
$$

Therefore,

$$
\lim _{\epsilon \rightarrow 0} \frac{C_{J}}{\epsilon} H_{\partial E}^{J_{\epsilon}}(0)=(N-1) H_{\partial E}(0)
$$

In the next example we will see that the assumption $\partial E$ is of class $C^{2}$ in the above result is necessary.

Example 3.8. Assume that $J(x)=\frac{1}{\left|B_{1}(0)\right|} \chi_{B_{1}(0)}$, with $B_{1}(0) \subset \mathbb{R}^{2}$. Let $\varphi: \mathbb{R} \rightarrow \mathbb{R}$ the function defined by

$$
\varphi(x):= \begin{cases}x^{2} & \text { if } x \geq 0 \\ -x^{2} & \text { if } x<0\end{cases}
$$

and consider the set

$$
E:=\left\{(x, y) \in \mathbb{R}^{2}: y<\varphi(x)\right\} .
$$

Then by symmetry we have $H_{\partial E}^{J_{\epsilon}}(0,0)=0$, and consequently

$$
\lim _{\epsilon \downarrow 0} \frac{C_{J}}{\epsilon} H_{\partial E}^{J_{\epsilon}}(0,0)=0 .
$$

Now, $H_{\partial E}(0,0)=2$ (here the curvature is understood as one over the largest radius of a tangent ball). Therefore, (3.2) is not true in this case.

\section{NONLOCAL OpERATORs. A CHARACTERIZATION OF NONLOCAL PERIMETER}

Following Gilboa-Osher [26] (see also [12]) we introduce the following nonlocal operators. For a function $u: \mathbb{R}^{N} \rightarrow \mathbb{R}$, we define its nonlocal gradient as the function $\nabla_{J} u: \mathbb{R}^{N} \times \mathbb{R}^{N} \rightarrow \mathbb{R}$ defined by

$$
\left(\nabla_{J} u\right)(x, y):=J(x-y)(u(y)-u(x)), \quad x, y \in \mathbb{R}^{N} .
$$

And for a function $\mathbf{z}: \mathbb{R}^{N} \times \mathbb{R}^{N} \rightarrow \mathbb{R}$, its nonlocal divergence $\operatorname{div}_{J} \mathbf{z}: \mathbb{R}^{N} \rightarrow \mathbb{R}$ is defined as

$$
\left(\operatorname{div}_{J} \mathbf{z}\right)(x):=\frac{1}{2} \int_{\mathbb{R}^{N}}(\mathbf{z}(x, y)-\mathbf{z}(y, x)) J(x-y) d y .
$$


Remark 4.1. A possible interpretation of $\operatorname{div}_{J} \mathbf{z}$ is the following. Suppose that $\mathbb{R}^{N}$ represents a continuous network, with a source uniformly distributed in $\Omega \subset \mathbb{R}^{N}$ and being $\mathbb{R}^{N} \backslash \Omega$ a sink. If the transportation activity is described by $\mathbf{z}$, in such a way that at each point $x \in \Omega, \mathbf{z}(x, y) J(x-y)$ is the incoming quantity of flow from $y \in \mathbb{R}^{N}$ and $\mathbf{z}(y, x) J(x-y)$ is the outcoming flow at $y \in \mathbb{R}^{N}$, then

$$
2\left(\operatorname{div}_{J} \mathbf{z}\right)(x):=\int_{\mathbb{R}^{N}}(\mathbf{z}(x, y)-\mathbf{z}(y, x)) J(x-y) d y,
$$

represents the total flow at $x$.

For $p \geq 1$, we define the space

$$
X_{J}^{p}\left(\mathbb{R}^{N}\right):=\left\{\mathbf{z} \in L^{\infty}\left(\mathbb{R}^{N} \times \mathbb{R}^{N}\right): \operatorname{div}_{J} \mathbf{z} \in L^{p}\left(\mathbb{R}^{N}\right)\right\} .
$$

Observe that $X_{J}^{\infty}\left(\mathbb{R}^{N}\right)=L^{\infty}\left(\mathbb{R}^{N} \times \mathbb{R}^{N}\right)$. For $u \in B V_{J}\left(\mathbb{R}^{N}\right) \cap L^{p^{\prime}}\left(\mathbb{R}^{N}\right)$ and $\mathbf{z} \in X_{J}^{p}\left(\mathbb{R}^{N}\right), 1 \leq p \leq \infty$, we have the following Green formula

$$
\int_{\mathbb{R}^{N}} u(x)\left(\operatorname{div}_{J} \mathbf{z}\right)(x) d x=-\frac{1}{2} \int_{\mathbb{R}^{N} \times \mathbb{R}^{N}}\left(\nabla_{J} u\right)(x, y) \mathbf{z}(x, y) d x d y .
$$

In the next result we characterize $\mathcal{F}_{J}$ and the nonlocal perimeter using the nonlocal divergence operator. Let us denote by $\operatorname{sign}_{0}(r)$ the usual sign function and by $\operatorname{sign}(r)$ the multivalued sign function for which $\operatorname{sign}(0)=[-1,1]$.

Proposition 4.2. Let $1 \leq p \leq \infty$. For $u \in B V_{J}\left(\mathbb{R}^{N}\right) \cap L^{p^{\prime}}\left(\mathbb{R}^{N}\right)$, we have

$$
\mathcal{F}_{J}(u)=\sup \left\{\int_{\mathbb{R}^{N}} u(x)\left(\operatorname{div}_{J} \mathbf{z}\right)(x) d x: \mathbf{z} \in X_{J}^{p}\left(\mathbb{R}^{N}\right),\|\mathbf{z}\|_{\infty} \leq 1\right\} .
$$

If $u \notin B V_{J}\left(\mathbb{R}^{N}\right)$ then $\sup \left\{\int_{\mathbb{R}^{N}} u(x)\left(\operatorname{div}_{J} \mathbf{z}\right)(x) d x: \mathbf{z} \in X_{J}^{p}\left(\mathbb{R}^{N}\right),\|\mathbf{z}\|_{\infty} \leq 1\right\}=+\infty$.

In particular, for any measurable set $E \subset \mathbb{R}^{N}$, we have

$$
P_{J}(E)=\sup \left\{\int_{E}\left(\operatorname{div}_{J} \mathbf{z}\right)(x) d x: \mathbf{z} \in X_{J}^{1}\left(\mathbb{R}^{N}\right),\|\mathbf{z}\|_{\infty} \leq 1\right\} .
$$

Proof. Let $u \in L^{1}\left(\mathbb{R}^{N}\right) \cap L^{p^{\prime}}\left(\mathbb{R}^{N}\right)$. Given $\mathbf{z} \in X_{J}^{p}\left(\mathbb{R}^{N}\right)$ with $\|\mathbf{z}\|_{\infty} \leq 1$, applying Green formula (4.1), we have

$$
\begin{gathered}
\int_{\mathbb{R}^{N}} u(x)\left(\operatorname{div}_{J} \mathbf{z}\right)(x) d x=\frac{1}{2} \int_{\mathbb{R}^{N} \times \mathbb{R}^{N}}\left(\nabla_{J} u\right)(x, y) \mathbf{z}(x, y) d x d y \\
\leq \frac{1}{2} \int_{\mathbb{R}^{N} \times \mathbb{R}^{N}}|u(y)-u(x)| J(x-y) d x d y=\mathcal{F}_{J}(u) .
\end{gathered}
$$

Therefore,

$$
\sup \left\{\int_{\mathbb{R}^{N}} u(x)\left(\operatorname{div}_{J} \mathbf{z}\right)(x) d x: \mathbf{z} \in X_{J}^{p}\left(\mathbb{R}^{N}\right),\|\mathbf{z}\|_{\infty} \leq 1\right\} \leq \mathcal{F}_{J}(u) .
$$


On the other hand, if we define $\mathbf{z}_{n}(x, y):=\operatorname{sign}_{0}(u(y)-u(x)) \chi_{B_{n}(0,0)}(x, y)$, then $\mathbf{z}_{n} \in X_{J}^{p}\left(\mathbb{R}^{N}\right)$ with $\left\|\mathbf{z}_{n}\right\|_{\infty} \leq 1$ and

$$
\begin{aligned}
\mathcal{F}_{J}(u) & =\frac{1}{2} \int_{\mathbb{R}^{N} \times \mathbb{R}^{N}}|u(y)-u(x)| J(x-y) d x d y=\lim _{n \rightarrow \infty} \frac{1}{2} \int_{B_{n}(0,0)}|u(y)-u(x)| J(x-y) d x d y \\
= & \lim _{n \rightarrow \infty} \frac{1}{2} \int_{\mathbb{R}^{N} \times \mathbb{R}^{N}}\left(\nabla_{J} u\right)(x, y) \mathbf{z}_{n}(x, y) d x d y=\lim _{n \rightarrow \infty} \int_{\mathbb{R}^{N}} u(x)\left(\operatorname{div}_{J} \mathbf{z}_{n}\right)(x) d x \\
\leq & \sup \left\{\int_{\mathbb{R}^{N}} u(x)\left(\operatorname{div}_{J} \mathbf{z}\right)(x) d x: \mathbf{z} \in X_{J}^{p}\left(\mathbb{R}^{N}\right),\|\mathbf{z}\|_{\infty} \leq 1\right\} .
\end{aligned}
$$

Remark 4.3. Observe that for $u \in L^{1}\left(\mathbb{R}^{N}\right)$ we are saying that

$$
\mathcal{F}_{J}(u)=\sup \left\{\int_{\mathbb{R}^{N}} u(x)\left(\operatorname{div}_{J} \mathbf{z}\right)(x) d x: \mathbf{z} \in L^{\infty}\left(\mathbb{R}^{N} \times \mathbb{R}^{N}\right),\|\mathbf{z}\|_{\infty} \leq 1\right\} .
$$

In particular, for any measurable set $E \subset \mathbb{R}^{N}$ with finite measure, we have

$$
P_{J}(E)=\sup \left\{\int_{E}\left(\operatorname{div}_{J} \mathbf{z}\right)(x) d x: \mathbf{z} \in L^{\infty}\left(\mathbb{R}^{N} \times \mathbb{R}^{N}\right),\|\mathbf{z}\|_{\infty} \leq 1\right\} .
$$

\section{The NONLOCAL 1-LaPlacian}

Nonlocal evolution equations of the form

$$
u_{t}(x, t)=\int_{\mathbb{R}^{N}} J(x-y)|u(y, t)-u(x, t)|^{p-2}(u(y, t)-u(x, t)) d y, \quad p \geq 1
$$

and variations of it, have been recently widely used to model diffusion processes (see [11] and the references therein).

For $p=1$, using the nonlocal calculus from Section 4, we have formally

$$
\begin{aligned}
\operatorname{div}_{J} & \left(\frac{\nabla_{J} u}{\left|\nabla_{J} u\right|}\right)(x)=\frac{1}{2} \int_{\mathbb{R}^{N}} J(x-y)\left(\frac{\nabla_{J} u}{\left|\nabla_{J} u\right|}(x, y)-\frac{\nabla_{J} u}{\left|\nabla_{J} u\right|}(y, x)\right) d y \\
\quad= & \frac{1}{2} \int_{\mathbb{R}^{N}} J(x-y)\left(\frac{(u(y)-u(x)) J(x-y)-(u(x)-u(y)) J(x-y)}{J(x-y)|u(y)-u(x)|}\right) d y \\
\quad= & \int_{\mathbb{R}^{N}} J(x-y) \frac{u(y)-u(x)}{|u(y)-u(x)|} d y,
\end{aligned}
$$

that we have called nonlocal 1-Laplacian operator in [8] and [9]:

$$
\Delta_{1}^{J} u(x):=\int_{\mathbb{R}^{N}} J(x-y) \frac{u(y)-u(x)}{|u(y)-u(x)|} d y \quad \text { for } u \in L^{1}\left(\mathbb{R}^{N}\right), x \in \mathbb{R}^{N} .
$$

Also, if $p>1$, we have

$$
\operatorname{div}_{J^{1 / p}}\left(\left|\nabla_{J^{1 / p}} u\right|^{p-2} \nabla_{J^{1 / p}} u\right)(x)=\int_{\mathbb{R}^{N}} J(x-y)|u(y)-u(x)|^{p-2}(u(y)-u(x)) d y,
$$

that we have called nonlocal p-Laplacian operator. In [8] and [9] we have studied these nonlocal operators with different boundary conditions, from these works we take the following definition. 
Definition 5.1. Given $v \in L^{1}\left(\mathbb{R}^{N}\right)$ we say that $u \in L^{1}\left(\mathbb{R}^{N}\right)$ is a solution of

$$
-\Delta_{1}^{J} u \ni v \text { in } \mathbb{R}^{N}
$$

if there exists $\mathbf{g} \in L^{\infty}\left(\mathbb{R}^{N} \times \mathbb{R}^{N}\right)$ with $\|g\|_{\infty} \leq 1$ verifying

$$
\begin{gathered}
\mathbf{g}(x, y)=-\mathbf{g}(y, x) \quad \text { for }(x, y) \text { a.e in } \mathbb{R}^{N} \times \mathbb{R}^{N}, \\
J(x-y) \mathbf{g}(x, y) \in J(x-y) \operatorname{sign}(u(y)-u(x)) \quad \text { a.e }(x, y) \in \mathbb{R}^{N} \times \mathbb{R}^{N},
\end{gathered}
$$

and

$$
-\int_{\mathbb{R}^{N}} J(x-y) \mathbf{g}(x, y) d y=v(x) \quad \text { a.e } x \in \mathbb{R}^{N} .
$$

We point out that, in general, the operator $\Delta_{1}^{J}$ is multivalued (see Remark 6.8).

In order to study the Cauchy problem associated with the nonlocal 1-Laplacian, we will see that we can consider it as the gradient flow in $L^{2}\left(\mathbb{R}^{N}\right)$ of the functional $\mathcal{F}_{J}$. For that we consider now the functional $\left.\mathcal{F}_{1}^{J}: L^{2}\left(\mathbb{R}^{N}\right) \rightarrow\right]-\infty,+\infty$ ] defined by

$$
\mathcal{F}_{1}^{J}(u):= \begin{cases}\mathcal{F}_{J}(u) & \text { if } u \in L^{2}\left(\mathbb{R}^{N}\right) \cap B V_{J}\left(\mathbb{R}^{N}\right), \\ +\infty & \text { if } u \in L^{2}\left(\mathbb{R}^{N}\right) \backslash B V_{J}\left(\mathbb{R}^{N}\right),\end{cases}
$$

which is convex and lower semi-continuous. Following the method used in [7] to get the characterization of the subdifferential of the total variation, we get the following characterization of the subdifferential of the functional $\mathcal{F}_{1}^{J}$.

Given a functional $\Phi: L^{2}\left(\mathbb{R}^{N}\right) \rightarrow[0, \infty]$, we define $\widetilde{\Phi}: L^{2}\left(\mathbb{R}^{N}\right) \rightarrow[0, \infty]$ as

$$
\widetilde{\Phi}(v):=\sup \left\{\frac{\int_{\mathbb{R}^{N}} v(x) w(x) d x}{\Phi(w)}: w \in L^{2}\left(\mathbb{R}^{N}\right)\right\}
$$

with the convention that $\frac{0}{0}=\frac{0}{\infty}=0$. Obviously, if $\Phi_{1} \leq \Phi_{2}$, then $\widetilde{\Phi}_{2} \leq \widetilde{\Phi}_{1}$.

Theorem 5.2. Let $u \in L^{1}\left(\mathbb{R}^{N}\right) \cap L^{2}\left(\mathbb{R}^{N}\right)$ and $v \in L^{2}\left(\mathbb{R}^{N}\right)$. The following assertions are equivalent:

(i) $v \in \partial \mathcal{F}_{1}^{J}(u)$;

(ii) there exists $\mathbf{z} \in X_{J}^{2}\left(\mathbb{R}^{N}\right),\|\mathbf{z}\|_{\infty} \leq 1$ such that

$$
v=-\operatorname{div}_{J} \mathbf{z}
$$

and

$$
\int_{\mathbb{R}^{N}} u(x) v(x) d x=\mathcal{F}_{1}^{J}(u)
$$

(iii) there exists $\mathbf{z} \in X_{J}^{2}\left(\mathbb{R}^{N}\right),\|\mathbf{z}\|_{\infty} \leq 1$ such that (5.1) hold and

$$
\mathcal{F}_{1}^{J}(u)=\frac{1}{2} \int_{\mathbb{R}^{N} \times \mathbb{R}^{N}} \nabla_{J} u(x, y) \mathbf{z}(x, y) d x d y ;
$$


(iv) $-\Delta_{1}^{J} u \ni v$ in $\mathbb{R}^{N}$;

(v) there exists $\mathbf{g} \in L^{\infty}\left(\mathbb{R}^{N} \times \mathbb{R}^{N}\right)$ antisymmetric with $\|\mathbf{g}\|_{\infty} \leq 1$ such that

$$
-\int_{\mathbb{R}^{N}} J(x-y) \mathbf{g}(x, y) d y=v(x) \quad \text { a.e } x \in \mathbb{R}^{N},
$$

and

$$
-\int_{\mathbb{R}^{N}} \int_{\mathbb{R}^{N}} J(x-y) \mathbf{g}(x, y) d y u(x) d x=\mathcal{F}_{1}^{J}(u) .
$$

Proof. Since $\mathcal{F}_{1}^{J}$ is convex, lower semi-continuous and positive homogeneous of degree 1 , by [7, Theorem 1.8], we have

$$
\partial \mathcal{F}_{1}^{J}(u)=\left\{v \in L^{2}\left(\mathbb{R}^{N}\right): \widetilde{\mathcal{F}_{1}^{J}}(v) \leq 1, \int_{\mathbb{R}^{N}} u(x) v(x) d x=\mathcal{F}_{1}^{J}(u)\right\} .
$$

We define, for $v \in L^{2}\left(\mathbb{R}^{N}\right)$,

$$
\Psi(v):=\inf \left\{\|\mathbf{z}\|_{\infty}: \mathbf{z} \in X_{J}^{2}\left(\mathbb{R}^{N}\right), v=-\operatorname{div}_{J} \mathbf{z}\right\} .
$$

Observe that $\Psi$ is convex, lower semi-continuous and positive homogeneous of degree 1 . Moreover, it is easy to see that, if $\Psi(v)<\infty$, the infimum in (5.4) is attained i.e., there exists some $\mathbf{z} \in X_{J}^{2}\left(\mathbb{R}^{N}\right)$, $v=-\operatorname{div}_{J} \mathbf{z}$ and $\Psi(v)=\|\mathbf{z}\|_{\infty}$.

Let us see that

$$
\Psi=\widetilde{\mathcal{F}_{1}^{J}}
$$

If $\Psi(v)=\infty$, then we have $\widetilde{\mathcal{F}_{1}^{J}}(v) \leq \Psi(v)$. Thus, we may assume that $\Psi(v)<\infty$. Let $\mathbf{z} \in L^{\infty}\left(\mathbb{R}^{N} \times \mathbb{R}^{N}\right)$ such that $v=-\operatorname{div}_{J} \mathbf{z}$. Then, for $w \in L^{2}\left(\mathbb{R}^{N}\right)$, we have

$$
\int_{\mathbb{R}^{N}} w(x) v(x) d x=\frac{1}{2} \int_{\mathbb{R}^{N} \times \mathbb{R}^{N}}\left(\nabla_{J} w\right)(x, y) \mathbf{z}(x, y) d x d y \leq\|\mathbf{z}\|_{\infty} \mathcal{F}_{1}^{J}(w) .
$$

Taking supremun in $w$ we obtain that $\widetilde{\mathcal{F}_{1}^{J}}(v) \leq\|\mathbf{z}\|_{\infty}$. Now, taking infimun in $\mathbf{z}$, we get $\widetilde{\mathcal{F}_{1}^{J}}(v) \leq \Psi(v)$.

To prove the opposite inequality, let us denote

$$
D:=\left\{\operatorname{div}_{J} \mathbf{z}: \mathbf{z} \in X_{J}^{2}\left(\mathbb{R}^{N}\right)\right\} .
$$

Then, by (4.2), we have, for $v \in L^{2}\left(\mathbb{R}^{N}\right)$,

$$
\begin{aligned}
\widetilde{\Psi}(v) & =\sup \left\{\frac{\int_{\mathbb{R}^{N}} w(x) v(x) d x}{\Psi(w)}: w \in L^{2}\left(\mathbb{R}^{N}\right)\right\} \geq \sup \left\{\frac{\int_{\mathbb{R}^{N}} w(x) v(x) d x}{\Psi(w)}: w \in D\right\} \\
& =\sup \left\{\frac{\int_{\mathbb{R}^{N}} \operatorname{div}_{J} \mathbf{Z}(x) v(x) d x}{\|\mathbf{z}\|_{\infty}}: \mathbf{z} \in X_{J}^{2}\left(\mathbb{R}^{N}\right)\right\}=\mathcal{F}_{1}^{J}(v) .
\end{aligned}
$$


Observe that the last term is equal to $+\infty$ if $v \in L^{2}\left(\mathbb{R}^{N}\right) \backslash B V_{J}\left(\mathbb{R}^{N}\right)$. Thus, $\mathcal{F}_{1}^{J} \leq \widetilde{\Psi}$, which implies by $\left[7\right.$, Proposition 1.6], that $\Psi=\widetilde{\widetilde{\Psi}} \leq \widetilde{\mathcal{F}_{1}^{J}}$. Therefore, $\Psi=\widetilde{\mathcal{F}_{1}^{J}}$, and consequently from (5.3), we get

$$
\begin{aligned}
& \partial \mathcal{F}_{1}^{J}(u)=\left\{v \in L^{2}\left(\mathbb{R}^{N}\right): \Psi(v) \leq 1, \int_{\mathbb{R}^{N}} u(x) v(x) d x=\mathcal{F}_{1}^{J}(u)\right\} \\
& \quad=\left\{v \in L^{2}\left(\mathbb{R}^{N}\right): \exists \mathbf{z} \in X_{J}^{2}\left(\mathbb{R}^{N}\right), v=-\operatorname{div}_{J} \mathbf{z},\|\mathbf{z}\|_{\infty} \leq 1, \int_{\mathbb{R}^{N}} u(x) v(x) d x=\mathcal{F}_{1}^{J}(u)\right\},
\end{aligned}
$$

from where it follows the equivalence between (i) and (ii).

To get the equivalence between (ii) and (iii) we only need to apply the Green formula (4.1).

By the antisimetry of $\mathbf{g}$ it is easy to see that (iv) and (v) are equivalent. On the other hand, to see that (iii) imply (v), it is enough to take $\mathbf{g}(x, y)=\frac{1}{2}(\mathbf{z}(x, y)-\mathbf{z}(y, x))$. Finally, to see that (v) imply (ii), it is enough to take $\mathbf{z}(x, y)=\mathbf{g}(x, y)$ (observe that, from $(5.2),-\operatorname{div}_{J}(\mathbf{g})=v$, so $\mathbf{g} \in X_{J}^{2}\left(\mathbb{R}^{N}\right)$ ).

Remark 5.3. Observe that if $v \in \partial \mathcal{F}_{1}^{J}(u)$, then any function $\mathbf{z}$ satisfying the characterization of Theorem 5.2 also satisfies

$$
J(x-y) \mathbf{z}(x, y) \in J(x-y) \operatorname{sign}\left((u(y)-u(x)) \quad \text { a.e. in } \mathbb{R}^{N} \times \mathbb{R}^{N} .\right.
$$

But, moreover, we can choose one being antisymmetric.

By Theorem 5.2 and following [11, Theorem 7.5] it is easy to prove the following result.

Lemma 5.4. $\partial \mathcal{F}_{1}^{J}$ is a $m$-completely accretive operator in $L^{2}\left(\mathbb{R}^{N}\right)$.

As consequence of Theorem 5.2 and Lemma 5.4 we can give the following existence and uniqueness result for the Cauchy problem

$$
\begin{cases}u_{t}-\Delta_{1}^{J} u \ni 0 & \text { in }(0, T) \times \mathbb{R}^{N} \\ u(0, x)=u_{0}(x) & x \in \mathbb{R}^{N} .\end{cases}
$$

Theorem 5.5. For every $u_{0} \in L^{2}\left(\mathbb{R}^{N}\right)$ there exists a unique solution of the Cauchy problem (5.5) in $(0, T)$ for any $T>0$, in the following sense: $u \in W^{1,1}\left(0, T ; L^{2}\left(\mathbb{R}^{N}\right)\right), u(0, \cdot)=u_{0}$, and for almost all $t \in(0, T)$

$$
u_{t}(t, \cdot)-\Delta_{1}^{J} u(t) \ni 0 .
$$

Moreover, we have the following contraction principle in any $L^{q}\left(\mathbb{R}^{N}\right)$-space, $1 \leq q \leq \infty$ :

$$
\|u(t)-v(t)\|_{q} \leq\left\|u_{0}-v_{0}\right\|_{q} \quad \forall 0<t<T,
$$

for any pair of solutions, $u, v$, of problem (5.5) with initial data $u_{0}, v_{0}$ respectively.

Proof. By the theory of maximal monotone operators (see [15]), and having in mind the characterization of the subdifferential of $\mathcal{F}_{1}^{J}$, for every $u_{0} \in L^{2}(\Omega)$ there exists a unique strong solution of the abstract Cauchy problem

$$
\left\{\begin{array}{l}
u^{\prime}(t)+\partial \mathcal{F}_{1}^{J}(u(t) \ni 0, \quad t \in(0, T), \\
u(0)=u_{0}
\end{array}\right.
$$

that is exactly the concept of solution given. The contraction principle is consequence of being the operator completely accretive. 


\section{Nonlocal Cheeger and Calibrable sets}

For any measurable set $E \subset \mathbb{R}^{N}$ with $|E|>0$, we define

$$
\lambda_{E}^{J}:=\frac{P_{J}(E)}{|E|} .
$$

As a consequence of (2.1), we have that $\lambda_{E}^{J} \leq 1$.

Given a non-null, measurable and bounded set $\Omega \subset \mathbb{R}^{N}$ we define its $J$-Cheeger constant by

$$
h_{1}^{J}(\Omega):=\inf \left\{\lambda_{E}^{J}: E \subset \Omega, E \text { measurable with }|E|>0\right\} .
$$

We have that $h_{1}^{J}(\Omega) \leq 1$. A measurable set $E_{\Omega} \subset \Omega$ achieving the infimun in (6.1) is said to be a $J$-Cheeger set of $\Omega$. Also we say that $\Omega$ is $J$-calibrable if it is a $J$-Cheeger set of itself, that is, if $\Omega$ is a non-null measurable bounded set and

$$
h_{1}^{J}(\Omega)=\frac{P_{J}(\Omega)}{|\Omega|} .
$$

As a consequence of the Poincaré type inequality given in [11, Proposition 6.25], in the case that $J$ is continuous (the continuity of $J$ is not needed in what follows but only at this point) and radial and $\Omega$ is a bounded domain, we have that there exists a positive constant $0<\lambda=\lambda(J, \Omega)$ such that

$$
\lambda \int_{\mathbb{R}^{N}}|u(x)| d x \leq \int_{\mathbb{R}^{N}} \int_{\mathbb{R}^{N}} J(x-y)|u(y)-u(x)| d x d y
$$

for all $u \in L^{1}\left(\mathbb{R}^{N}\right)$ such that $u=0$ in $\mathbb{R}^{N} \backslash \Omega$. Then,

$$
\lambda|E| \leq 2 P_{J}(E) \text { for all measurable } E \subset \Omega,
$$

and consequently $h_{1}^{J}(\Omega) \geq \frac{\lambda}{2}$.

Let us now define

$$
\begin{aligned}
& \lambda_{1}^{J}(\Omega):=\inf \left\{\mathcal{F}_{J}(u): u \in B V_{J}\left(\mathbb{R}^{N}\right), u=0 \text { in } \mathbb{R}^{N} \backslash \Omega,\|u\|_{1}=1\right\} \\
& =\inf \left\{\frac{\mathcal{F}_{J}(u)}{\|u\|_{1}}: u \in B V_{J}\left(\mathbb{R}^{N}\right), u=0 \text { in } \mathbb{R}^{N} \backslash \Omega, u \neq 0\right\} .
\end{aligned}
$$

Note that by the lack of compactness it is not clear if we can change inf by min.

It is well known (see [23]) that the classical Cheeger constant

$$
h_{1}(\Omega):=\inf \left\{\frac{\operatorname{Per}(E)}{|E|}: E \subset \Omega,|E|>0\right\},
$$

for $\Omega$ a bounded smooth domain, is an optimal Poincaré constant, namely, it coincides with the first eigenvalue of the 1-Laplacian:

$$
h_{1}(\Omega)=\lambda_{1}(\Omega):=\inf \left\{\frac{\int_{\Omega}|D u|+\int_{\partial \Omega}|u| d \mathcal{H}^{N-1}}{\|u\|_{L^{1}(\Omega)}}: u \in B V(\Omega), u \neq 0\right\} .
$$

It is also characterized (see [25]) as

$$
h_{1}(\Omega)=\sup \left\{h \in \mathbb{R}: \exists V \in L^{\infty}\left(\Omega, \mathbb{R}^{N}\right),\|V\|_{\infty} \leq 1, \operatorname{div} V \geq h\right\},
$$

which is usually referred to as a continuous version of the Min Cut Max Flow Theorem. In the next result we obtain nonlocal versions of these two characterizations of the $J$-Cheeger constant given in (6.1). 
Theorem 6.1. Let $\Omega$ be a non-null, measurable and bounded set of $\mathbb{R}^{N}$, then

$$
h_{1}^{J}(\Omega)=\lambda_{1}^{J}(\Omega)
$$

and

$$
\begin{aligned}
h_{1}^{J}(\Omega) & =\sup \left\{h \in \mathbb{R}^{+}: \exists \mathbf{z} \in X_{J}^{\infty}\left(\mathbb{R}^{N}\right),\|\mathbf{z}\|_{\infty} \leq 1, \operatorname{div}_{J} \mathbf{z} \geq h \text { in } \Omega\right\} \\
& =\sup \left\{\frac{1}{\|\mathbf{z}\|_{\infty}}: \operatorname{div}_{J} \mathbf{z}=\chi_{\Omega}\right\} \\
& =\sup \left\{\frac{1}{\|\mathbf{z}\|_{\infty}}: \operatorname{div}_{J} \mathbf{z}=1 \text { in } \Omega\right\} .
\end{aligned}
$$

Proof. Given a measurable subset $E \subset \Omega$ with $|E|>0$, we have

$$
\frac{\mathcal{F}_{J}\left(\chi_{E}\right)}{\left\|\chi_{E}\right\|_{1}}=\frac{P_{J}(E)}{|E|} .
$$

Therefore, $\lambda_{1}^{J}(\Omega) \leq h_{1}^{J}(\Omega)$. On the other hand, by the coarea formula (2.4) and Cavalieri formula, given $u \in B V_{J}\left(\mathbb{R}^{N}\right)$, with $u=0$ in $\mathbb{R}^{N} \backslash \Omega$ and $u \neq 0$, we have

$$
\frac{\mathcal{F}_{J}(u)}{\|u\|_{1}}=\frac{\int_{-\infty}^{+\infty} P_{J}\left(E_{t}(u)\right) d t}{\int_{-\infty}^{+\infty}\left|E_{t}(u)\right| d t} \geq h_{1}^{J}(\Omega)
$$

and taking infimum we get $\lambda_{1}^{J}(\Omega) \geq h_{1}^{J}(\Omega)$. Therefore (6.2) holds true.

Let

$$
A:=\left\{h \in \mathbb{R}^{+}: \exists \mathbf{z} \in X_{J}^{\infty}\left(\mathbb{R}^{N}\right),\|\mathbf{z}\|_{\infty} \leq 1, \operatorname{div}_{J} \mathbf{z} \geq h \text { in } \Omega\right\}
$$

and $\alpha:=\sup A$ (observe that $0 \leq \alpha \leq 1$ ). Given $h \in A$ and $E \subset \Omega$ with $|E|>0$, applying (4.3), we get

$$
h|E|=\int_{E} h d x \leq \int_{E} \operatorname{div}_{J} \mathbf{z}(x) d x \leq P_{J}(E) .
$$

Hence,

$$
h \leq \frac{P_{J}(E)}{|E|},
$$

and, taking supremum in $h$ and infimum in $E$, we obtain that $\alpha \leq h_{1}^{J}(\Omega)$.

By (6.2), we have

$$
\begin{aligned}
\frac{1}{h_{1}^{J}(\Omega)} & =\sup \left\{\frac{\|u\|_{1}}{\mathcal{F}_{J}(u)}: u \in B V_{J}\left(\mathbb{R}^{N}\right), u=0 \text { in } \mathbb{R}^{N} \backslash \Omega, u \neq 0\right\} \\
& =\sup \left\{\frac{\|u\|_{1}}{\mathcal{F}_{J}(u)}: u \in B V_{J}\left(\mathbb{R}^{N}\right), u \geq 0, u=0 \text { in } \mathbb{R}^{N} \backslash \Omega, u \neq 0\right\} \\
& =\sup \left\{\int_{\Omega} u(x) d x: \mathcal{F}_{J}(u) \leq 1, u \in B V_{J}\left(\mathbb{R}^{N}\right), u=0 \text { in } \mathbb{R}^{N} \backslash \Omega\right\} \\
& =\sup \left\{\left\langle u, \chi_{\Omega}\right\rangle-\Xi(L(u)): u \in L^{1}\left(\mathbb{R}^{N}\right)\right\},
\end{aligned}
$$


being $L: L^{1}\left(\mathbb{R}^{N}\right) \rightarrow L^{1}\left(\mathbb{R}^{N} \times \mathbb{R}^{N}\right)$ the linear map

$$
L(u)(x, y):=\frac{1}{2}(u(x)-u(y)) J(x-y),
$$

and $\Xi: L^{1}\left(\mathbb{R}^{N} \times \mathbb{R}^{N}\right) \rightarrow[0,+\infty]$ the convex function

$$
\Xi(w):= \begin{cases}0 & \text { if }\|w\|_{L^{1}\left(\mathbb{R}^{N} \times \mathbb{R}^{N}\right)} \leq 1, \\ +\infty & \text { otherwise. }\end{cases}
$$

By the Frenchel-Rockafeller duality Theorem ([16, Th. 1.12]) and having in mind [22, Prop. 5], we have

$$
\sup \left\{\left\langle u, \chi_{\Omega}\right\rangle-\Xi(L(u)): u \in L^{1}\left(\mathbb{R}^{N}\right)\right\}=\inf \left\{\Xi^{*}(\mathbf{z}): L^{*}(\mathbf{z})=\chi_{\Omega}\right\} .
$$

Now,

$$
\Xi^{*}(\mathbf{z})=\sup \left\{\int_{\mathbb{R}^{N} \times \mathbb{R}^{N}} \mathbf{z}(x, y) w(x, y) d x d y-\Xi(w): w \in L^{1}\left(\mathbb{R}^{N} \times \mathbb{R}^{N}\right)\right\}=\|\mathbf{z}\|_{\infty} .
$$

On the other hand,

$$
\begin{gathered}
\left\langle L^{*}(\mathbf{z}), u\right\rangle=\langle\mathbf{z}, L(u)\rangle=\int_{\mathbb{R}^{N} \times \mathbb{R}^{N}} \mathbf{z}(x, y) \frac{1}{2}(u(x)-u(y)) J(x-y) d x d y \\
=\frac{1}{2} \int_{\mathbb{R}^{N} \times \mathbb{R}^{N}}(\mathbf{z}(x, y)-\mathbf{z}(y, x)) J(x-y) u(x) d x d y=\left\langle\operatorname{div}_{J} \mathbf{z}, u\right\rangle,
\end{gathered}
$$

that is, $L^{*}(\mathbf{z})=\operatorname{div}_{J} \mathbf{z}$. Consequently,

$$
\frac{1}{h_{1}^{J}(\Omega)}=\inf \left\{\|\mathbf{z}\|_{\infty}: \operatorname{div}_{J} \mathbf{z}=\chi_{\Omega}\right\}
$$

from where it follows that

$$
h_{1}^{J}(\Omega)=\sup \left\{\frac{1}{\|\mathbf{z}\|_{\infty}}: \operatorname{div}_{J} \mathbf{z}=\chi_{\Omega}\right\} \leq \sup \left\{\frac{1}{\|\mathbf{z}\|_{\infty}}: \operatorname{div}_{J} \mathbf{z}=1 \text { in } \Omega\right\} \leq \alpha,
$$

and we finish the proof of (6.3).

Remark 6.2. It is well know that every bounded domain $\Omega \subset \mathbb{R}^{N}$ with Lipschitz boundary contains a classical Cheeger set $E$, that is, a set $E \subset \Omega$ such that

$$
h_{1}(\Omega)=\frac{\operatorname{Per}(E)}{|E|} .
$$

Furthermore, in [3] it is proved that there is a unique Cheeger set inside any non-trivial convex body in $\mathbb{R}^{N}$, being this Cheeger set convex.

On the other hand, in [14] it is proved that for any $s \in(0,1)$, every open and bounded set $\Omega \subset \mathbb{R}^{N}$ admits and $s$-Cheeger set, that is, a set $E \subset \Omega$ such that

$$
\frac{P_{s}(E)}{|E|}=\inf \left\{\frac{P_{s}(F)}{|F|}: F \subset \Omega,|F|>0\right\} .
$$

But we will show in Remark 6.11 that there are convex sets without a $J$-Cheeger set. This is due to the lack of compactness which is consequence to the fact that we are considering non-singular kernels.

As a consequence of the Isoperimetric Inequality (Theorem 2.4), we show that any ball is $J$-calibrable.

Proposition 6.3. Let $J$ be a nonnegative radially nonincreasing function. Then, any ball $B_{R}\left(x_{0}\right) \subset \mathbb{R}^{N}$ is $J$-calibrable. 
Proof. We can take $x_{0}=0$ as the center of the ball. First we prove that a ball $B_{R}(0) \subset \mathbb{R}^{N}$ is $J$-calibrable if and only if the function

verifies that

$$
\Theta(r)=\lambda_{B_{r}(0)}^{J}=\frac{P_{J}\left(B_{r}(0)\right)}{\left|B_{r}(0)\right|}
$$

$$
\Theta(r) \geq \Theta(R), \quad \forall r \in(0, R) .
$$

Obviously, the condition is necessary. On the other hand, given $E \subset B_{R}\left(x_{0}\right)$, by the isoperimetric inequality we have that

$$
\frac{P_{J}(E)}{|E|} \geq \frac{P_{J}\left(B_{r}(0)\right)}{\left|B_{r}(0)\right|}=\Theta(r)
$$

where $B_{r}(0)$ is a ball such that $\left|B_{r}(0)\right|=|E|$. Hence, since we are assuming that the function $\Theta(r)$ verifies (6.4), we have

$$
\frac{P_{J}(E)}{|E|} \geq \Theta(r) \geq \Theta(R)=\frac{P_{J}\left(B_{R}(0)\right)}{\left|B_{R}(0)\right|},
$$

and consequently $B_{R}(0)$ is $J$-calibrable.

By the above characterization we need to show that (6.4) holds. In fact, from (1.2),

$$
P_{J}\left(B_{r}(0)\right)=\left|B_{r}(0)\right|-\int_{B_{r}(0)} \int_{B_{r}(0)} J(x-y) d y d x .
$$

Hence (6.4) is true if and only if

$$
F(r) \leq F(R) \quad \text { for every } 0<r<R
$$

where

$$
F(r):=\frac{1}{\left|B_{r}(0)\right|} \int_{B_{r}(0)}\left(\int_{B_{r}(0)} J(x-y) d y\right) d x .
$$

Take $0<r<R$. Then, changing variables $z=\frac{R}{r} x$, we have

$$
\begin{gathered}
F(r)=\frac{1}{\left|B_{r}(0)\right|} \int_{B_{r}(0)}\left(\int_{B_{r}(0)} J(x-y) d y\right) d x=\frac{1}{\left|B_{R}(0)\right|} \int_{B_{R}(0)}\left(\int_{B_{r}(0)} J\left(\frac{r}{R} z-y\right) d y\right) d z \\
\leq \frac{1}{\left|B_{R}(0)\right|} \int_{B_{R}(0)}\left(\int_{B_{R}(0)} J(z-y) d y\right) d z=F(R),
\end{gathered}
$$

since, for any $z \in B_{R}(0), B_{r}\left(\frac{r}{R} z\right) \subset B_{R}(z)$, which implies

$$
\int_{B_{r}(0)} J\left(\frac{r}{R} z-y\right) d y=\int_{B_{r}\left(\frac{r}{R} z\right)} J(y) d y \leq \int_{B_{R}(z)} J(y) d y=\int_{B_{R}(0)} J(z-y) d y .
$$

Remark 6.4. For $J$ radially nonincreasing we have that $\Theta$ is indeed continuously differentiable in $] 0,+\infty[$. A simple calculation shows that

$$
\frac{d}{d r} P_{J}\left(B_{r}(0)\right)=H_{\partial B_{r}(0)}^{J}(y) \operatorname{Per}\left(B_{r}(0)\right),
$$


with $y \in \partial B_{r}(0)$. Then,

$$
\Theta^{\prime}(r)=\frac{H_{\partial B_{r}(0)}^{J}(y) \operatorname{Per}\left(B_{r}(0)\right)\left|B_{r}(0)\right|-\operatorname{Per}\left(B_{r}(0)\right) P_{J}\left(B_{r}(0)\right)}{\left|B_{r}(0)\right|^{2}},
$$

with $y \in \partial B_{r}(0)$ arbitrary.

Therefore, by the characterization given in the proof of Proposition 6.3 and having in mind that clearly $\operatorname{ess} \sup H_{\partial B_{r}(0)}^{J}(x)$ is attained on any point of the boundary of $B_{r}(0)$, we get $x \in B_{r}(0)$

$$
B_{r}(0) \text { is } J \text {-calibrable } \Longleftrightarrow \operatorname{ess~sup}_{x \in B_{r}(0)} H_{\partial B_{r}(0)}^{J}(x) \leq \lambda_{B_{r}(0)}^{J} .
$$

The next result shows that any measurable and non null set inside a ball of radius $\frac{1}{2}$ is $J$-calibrable when $J:=\frac{1}{\left|B_{1}(0)\right|} \chi_{B_{1}(0)}$.

Proposition 6.5. Let $J=\frac{1}{\left|B_{1}(0)\right|} \chi_{B_{1}(0)}$. If $\Omega \subset B_{\frac{1}{2}}(0)$ with $|\Omega|>0$, then $\Omega$ is J-calibrable.

Proof. Let $E \subset \Omega$ non null. For $x \in E$,

$$
E \subset B_{1}(x)
$$

Then,

and

$$
\begin{aligned}
P_{J}(E) & =\int_{E} \int_{\mathbb{R} \backslash E} J(x-y) d y d x=\frac{1}{\left|B_{1}(0)\right|} \int_{E} \int_{\mathbb{R}^{N} \backslash E} \chi_{B_{1}(0)}(x-y) d y d x \\
= & \frac{1}{\left|B_{1}(0)\right|} \int_{E} \int_{\mathbb{R}^{N} \backslash E} \chi_{B_{1}(x)}(y) d y d x=\frac{1}{\left|B_{1}(0)\right|} \int_{E}\left|B_{1}(x) \backslash E\right| d x \\
= & \frac{1}{\left|B_{1}(0)\right|} \int_{E}\left(\left|B_{1}(x)\right|-\left|E \cap B_{1}(x)\right|\right) d x=|E|\left(1-\frac{|E|}{\left|B_{1}(0)\right|}\right),
\end{aligned}
$$

$$
\frac{P_{J}(E)}{|E|}=\left(1-\frac{|E|}{\left|B_{1}(0)\right|}\right)
$$

that is decreasing with $|E|$. Hence the Cheeger constant of $\Omega$ is given by

$$
h_{1}^{J}(\Omega)=\left(1-\frac{|\Omega|}{\left|B_{1}(0)\right|}\right)=\frac{P_{J}(\Omega)}{|\Omega|},
$$

as we wanted to show.

Remark 6.6. For the local usual perimeter, when $\Omega$ is the union of two intervals in $\mathbb{R}, \Omega=(a, b) \cup(c, d)$, then the set is calibrable if and only if the two intervals have the same length (otherwise the Cheeger set inside $\Omega$ is the bigger interval). For the nonlocal perimeter with $J=\frac{1}{2} \chi_{[-1,1]}$ we have the following facts. Assume $c-b \geq 1$.

We want to compare $\frac{P_{J}(\Omega)}{|\Omega|}$ with the quotient of $\frac{P_{J}(E)}{|E|}$ for $E \subset(a, b) \cup(c, d)$. We decompose $E$ as $E=E_{1} \cup E_{2}$ with $E_{1}=E \cap(a, b)$ and $E_{2}=E \cap(c, d)$. For the case in which the two intervals that compose $\Omega$ have the same length we have

iff

$$
\frac{P_{J}(\Omega)}{|\Omega|} \leq \frac{P_{J}(E)}{|E|}
$$

$$
\frac{P_{J}((a, b))}{b-a} \leq \frac{P_{J}\left(E_{1}\right)+P_{J}\left(E_{2}\right)}{\left|E_{1}\right|+\left|E_{2}\right|},
$$


which is true since, if $\left|E_{i}\right| \neq 0$ then, by the Isoperimetric Inequality,

$$
\frac{P_{J}((a, b))}{b-a} \leq \frac{P_{J}\left(E_{i}\right)}{\left|E_{i}\right|} .
$$

On the other hand, if $b-a>d-c$ then

$$
\frac{P_{J}((a, b))}{|b-a|}<\frac{P_{J}(\Omega)}{|\Omega|}
$$

and therefore we have that $\Omega$ is not $J$-calibrable.

Let us recall that that, in the local case, a set $\Omega \subset \mathbb{R}^{N}$ is called calibrable if

$$
\frac{\operatorname{Per}(\Omega)}{|\Omega|}=\inf \left\{\frac{\operatorname{Per}(E)}{|E|}: E \subset \Omega, E \text { with finite perimeter, }|E|>0\right\} \text {. }
$$

In [2] it is proved the following characterization of convex calibrable set.

Theorem 6.7. ([2]) Given a bounded convex set $\Omega \subset \mathbb{R}^{N}$ of class $C^{1,1}$, the following facts are equivalent:

(a) $\Omega$ is calibrable.

(b) $\chi_{\Omega}$ satisfies $-\Delta_{1} \chi_{\Omega}=\frac{\operatorname{Per}(\Omega)}{|\Omega|} \chi_{\Omega}$, being $\Delta_{1} u:=\operatorname{div}\left(\frac{D u}{|D u|}\right)$.

When $\Omega$ is convex these statements are also equivalent to

(c) $(N-1) \underset{x \in \partial \Omega}{\operatorname{ess} \sup _{\partial \Omega}} H_{\partial \Omega}(x) \leq \frac{\operatorname{Per}(\Omega)}{|\Omega|}$.

We are going to study the validity of a similar result to the above theorem for the nonlocal case. In the following remark we will introduce the main idea that is behind the proof for the nonlocal case.

Remark 6.8. Let $\Omega \subset \mathbb{R}^{N}$ be a Borel set and assume there exists a constant $\lambda>0$ and a function $\tau$ with $\tau(x)=1$ in $\Omega$ such that

$$
-\lambda \tau \in \Delta_{1}^{J} \chi_{\Omega} \quad \text { in } \mathbb{R}^{N} .
$$

Then, there exists $\mathbf{g} \in L^{\infty}\left(\mathbb{R}^{N} \times \mathbb{R}^{N}\right), \mathbf{g}(x, y)=-\mathbf{g}(y, x)$ for almost all $(x, y) \in \mathbb{R}^{N} \times \mathbb{R}^{N},\|\mathbf{g}\|_{\infty} \leq 1$, satisfying

$$
\int_{\mathbb{R}^{N}} J(x-y) \mathbf{g}(x, y) d y=-\lambda \tau(x) \quad \text { a.e } x \in \mathbb{R}^{N} .
$$

with

$$
J(x-y) \mathbf{g}(x, y) \in J(x-y) \operatorname{sign}\left(\chi_{\Omega}(y)-\chi_{\Omega}(x)\right) \quad \text { a.e. }(x, y) \in \mathbb{R}^{N} \times \mathbb{R}^{N} .
$$

Then,

$$
\lambda|\Omega|=\int_{\mathbb{R}^{N}} \lambda \tau(x) \chi_{\Omega}(x) d x=-\int_{\mathbb{R}^{N}}\left(\int_{\mathbb{R}^{N}} J(x-y) \mathbf{g}(x, y) d y\right) \chi_{\Omega}(x) d x=\mathcal{F}_{J}\left(\chi_{\Omega}\right)=P_{J}(\Omega),
$$

and consequently

$$
\lambda=\lambda_{\Omega}^{J}:=\frac{P_{J}(\Omega)}{|\Omega|} .
$$

On the other hand, we observe again that the operator $\Delta_{1}^{J} \chi_{\Omega}$ is multivalued. Let us take, for example, $J:=\frac{1}{2} \chi_{[-1,1]}$. We have that

$$
-f \in \Delta_{1}^{J} \chi_{]-1,1[} \Longleftrightarrow \exists \mathbf{g} \text { antisymmetric, }\|\mathbf{g}\|_{\infty} \leq 1
$$


satisfying

$$
\int_{\mathbb{R}} J(x-y) \mathbf{g}(x, y) d y=-f(x) \quad \text { a.e } x \in \mathbb{R} .
$$

and

$$
J(x-y) \mathbf{g}(x, y) \in J(x-y) \operatorname{sign}\left(\chi_{[-1,1[}(y)-\chi_{]-1,1[}(x)\right) \quad \text { a.e. }(x, y) \in \mathbb{R} \times \mathbb{R} .
$$

Then, by taking for instance $\mathbf{g}(x, y)=\operatorname{sign}_{0}\left(\chi_{]-1,1}(y)-\chi_{]-1,1}(x)\right)$ we have, if $x \in[-1,1]$,

$$
f(x)=\frac{1}{2}|x|,
$$

and, if $x \notin[-1,1]$,

$$
f(x)= \begin{cases}-\frac{1}{2}(x+2) & \text { if }-2 \leq x \leq-1, \\ -\frac{1}{2}(2-x) & \text { if } 1 \leq x \leq 2, \\ 0 & \text { if } x \leq-2 \text { or } x \geq 2 .\end{cases}
$$

But, by taking

$$
\mathbf{g}(x, y)=\left\{\begin{aligned}
1 & \text { if } y \in[-1,1], x \notin[-1,1], \\
-1 & \text { if } x \in[-1,1], y \notin[-1,1], \\
\frac{1}{2} & \text { in }\{0<y<x<1\} \cup\{-1<x<y<0\} \cup\{1<y<x\} \cup\{y<x<-1\}, \\
-\frac{1}{2} & \text { in }\{0<x<y<1\} \cup\{-1<y<x<0\} \cup\{1<x<y\} \cup\{x<y<-1\}, \\
0 & \text { otherwise, }
\end{aligned}\right.
$$

we get a different but interesting representation for $\Delta_{1}^{J} \chi_{]-1,1[}$. We get that

$$
-\lambda_{]-1,1}^{J} \tau \in \Delta_{1}^{J} \chi_{]-1,1[} \quad \text { in } \mathbb{R}^{N}
$$

with

$$
\tau(x)= \begin{cases}1 & \text { if } x \in[-1,1], \\ -(|x|-2)^{-}, & \text {otherwhise }\end{cases}
$$

being the value of $\lambda_{]-1,1}^{J}$, as seen above, equal to $\frac{1}{4}$. Note that this function $\tau$ verifies that

$$
\tau=1 \text { in }]-1,1[\text {, }
$$

and this gives, as we will see in the next theorem, that $]-1,1[$ is $J$-calibrable. Of course, in this simple case, this was obtained previously by more elementary methods.

The next result is the nonlocal version of the fact that (a) is equivalent to (b) in Theorem 6.7.

Theorem 6.9. Let $\Omega \subset \mathbb{R}^{N}$ be a non-null measurable bounded set.

(i) Assume that $\int_{\Omega} J(x-y) d y>0$ for all $x \in \Omega$. If $\Omega$ is $J$-calibrable then there exists a function $\tau$ equal to 1 in $\Omega$ such that

$$
-\lambda_{\Omega}^{J} \tau \in \Delta_{1}^{J} \chi_{\Omega} \quad \text { in } \mathbb{R}^{N} .
$$

(ii) If there exists a function $\tau$ equal to 1 in $\Omega$ and satisfying (6.5) then $\Omega$ is $J$-calibrable. 
Proof. We first prove (ii): By hypothesis, there exists, $\mathbf{g}(x, y)=-\mathbf{g}(y, x)$ for almost all $(x, y) \in \mathbb{R}^{N} \times \mathbb{R}^{N}$, $\|\mathbf{g}\|_{\infty} \leq 1$, satisfying

$$
\int_{\mathbb{R}^{N}} J(x-y) \mathbf{g}(x, y) d y=-\lambda_{\Omega}^{J} \tau(x) \quad \text { a.e } x \in \mathbb{R}^{N} .
$$

with

$$
J(x-y) \mathbf{g}(x, y) \in J(x-y) \operatorname{sign}\left(\chi_{\Omega}(y)-\chi_{\Omega}(x)\right) \quad \text { a.e. }(x, y) \in \mathbb{R}^{N} \times \mathbb{R}^{N},
$$

and $\tau$ is a function such that

$$
\tau=1 \text { in } \Omega .
$$

Then, if $F$ is a bounded measurable set, $F \subset \Omega$, we have

$$
\begin{gathered}
\lambda_{\Omega}^{J}|F|=\lambda_{\Omega}^{J} \int_{\mathbb{R}^{N}} \tau(x) \chi_{F}(x) d x=-\int_{\mathbb{R}^{N}} \int_{\mathbb{R}^{N}} J(x-y) \mathbf{g}(x, y) \chi_{F}(x) d y d x \\
=\frac{1}{2} \int_{\mathbb{R}^{N}} \int_{\mathbb{R}^{N}} J(x-y) \mathbf{g}(x, y)\left(\chi_{F}(y)-\chi_{F}(x)\right) d y d x \leq P_{J}(F),
\end{gathered}
$$

Therefore, $h_{1}^{J}(\Omega)=\lambda_{\Omega}^{J}$, and consequently $\Omega$ is $J$-calibrable.

Let us now prove (i): Let $\tilde{\mathbf{g}} \in L^{\infty}\left(\mathbb{R}^{N} \times \mathbb{R}^{N}\right)$ be defined as

$$
\tilde{\mathbf{g}}(x, y):= \begin{cases}0 & \text { if } x \in \mathbb{R}^{N} \backslash \Omega, y \in \mathbb{R}^{N} \backslash \Omega, \\ -1 & \text { if } x \in \Omega, y \in \mathbb{R}^{N} \backslash \Omega, \\ 1 & \text { if } x \in \mathbb{R}^{N} \backslash \Omega, y \in \Omega, \\ \hat{g}(x) & \text { if } x, y \in \Omega,\end{cases}
$$

being $\hat{g}$ a function to be determined. We define

$$
\tau(x):=-\frac{1}{\lambda_{\Omega}^{J}} \int_{\mathbb{R}^{N}} J(x-y) \tilde{\mathbf{g}}(x, y) d y, \quad x \in \mathbb{R}^{N} .
$$

For $x \in \Omega$, we have

$$
\tau(x)=\frac{1}{\lambda_{\Omega}^{J}} \int_{\mathbb{R}^{N} \backslash \Omega} J(x-y) d y-\hat{g}(x) \frac{1}{\lambda_{\Omega}^{J}} \int_{\Omega} J(x-y) d y .
$$

Then, taking

$$
\hat{g}(x):=\frac{-\lambda_{\Omega}^{J}+\int_{\mathbb{R}^{N} \backslash \Omega} J(x-y) d y}{\int_{\Omega} J(x-y) d y}, \quad x \in \Omega,
$$

we have that $\tau(x)=1$ for all $x \in \Omega$. Moreover, for $x \in \mathbb{R}^{N} \backslash \Omega$,

$$
\tau(x)=-\frac{1}{\lambda_{\Omega}^{J}} \int_{\Omega} J(x-y) d y \leq 0 .
$$

We claim now that

$$
\lambda_{\Omega}^{J} \tau \in \partial \mathcal{F}_{1}^{J}(0) .
$$

Take $w \in L^{2}\left(\mathbb{R}^{N}\right)$ with $\mathcal{F}_{J}(w)<+\infty$. Since

$$
w(x)=\int_{0}^{\infty} \chi_{E_{t}(w)}(x) d t-\int_{-\infty}^{0}\left(1-\chi_{E_{t}(w)}\right)(x) d t,
$$


we have

$$
\begin{aligned}
\int_{\mathbb{R}^{N}} \lambda_{\Omega}^{J} \tau(x) w(x) d x=\int_{\mathbb{R}^{N}} \lambda_{\Omega}^{J} \tau(x)\left(\int_{-\infty}^{\infty} \chi_{E_{t}(w)}(x) d t\right) d x \\
=\lambda_{\Omega}^{J} \int_{-\infty}^{+\infty} \int_{\mathbb{R}^{N}} \tau(x) \chi_{E_{t}(w)}(x) d x d t .
\end{aligned}
$$

Now, using that $\Omega$ is $J$-calibrable we have that

$$
\begin{aligned}
& \lambda_{\Omega}^{J} \int_{-\infty}^{+\infty} \int_{\mathbb{R}^{N}} \tau(x) \chi_{E_{t}(w)}(x) d x d t=\lambda_{\Omega}^{J} \int_{-\infty}^{+\infty}\left|E_{t}(w) \cap \Omega\right| d t+\lambda_{\Omega}^{J} \int_{-\infty}^{+\infty} \int_{E_{t}(w) \backslash \Omega} \tau(x) d x d t \\
& \quad \leq \int_{-\infty}^{+\infty} P_{J}\left(E_{t}(w) \cap \Omega\right) d t+\lambda_{\Omega}^{J} \int_{-\infty}^{+\infty} \int_{E_{t}(w) \backslash \Omega} \tau(x) d x d t .
\end{aligned}
$$

By Proposition 2.2 and the coarea formula given in Theorem 2.6 we get

$$
\begin{aligned}
\int_{-\infty}^{+\infty} & P_{J}\left(E_{t}(w) \cap \Omega\right) d t+\lambda_{\Omega}^{J} \int_{-\infty}^{+\infty} \int_{E_{t}(w) \backslash \Omega} \tau(x) d x d t \\
= & \int_{-\infty}^{+\infty} P_{J}\left(E_{t}(w) \cap \Omega\right) d t+\int_{-\infty}^{+\infty} P_{J}\left(E_{t}(w) \backslash \Omega\right) d t-\int_{-\infty}^{+\infty} 2 L_{J}\left(E_{t}(w) \backslash \Omega, E_{t}(w) \cap \Omega\right) d t \\
& -\int_{-\infty}^{+\infty} P_{J}\left(E_{t}(w) \backslash \Omega\right) d t+\int_{-\infty}^{+\infty} 2 L_{J}\left(E_{t}(w) \backslash \Omega, E_{t}(w) \cap \Omega\right) d t+\lambda_{\Omega}^{J} \int_{-\infty}^{+\infty} \int_{E_{t}(w) \backslash \Omega} \tau(x) d x d t \\
= & \int_{-\infty}^{+\infty} P_{J}\left(E_{t}(w)\right) d t+I=\mathcal{F}_{J}(w)+I,
\end{aligned}
$$

with

$$
I:=-\int_{-\infty}^{+\infty} P_{J}\left(E_{t}(w) \backslash \Omega\right) d t+\int_{-\infty}^{+\infty} 2 L_{J}\left(E_{t}(w) \backslash \Omega, E_{t}(w) \cap \Omega\right) d t+\lambda_{\Omega}^{J} \int_{-\infty}^{+\infty} \int_{E_{t}(w) \backslash \Omega} \tau(x) d x d t
$$

Hence, if we prove that $I \leq 0$, we get

$$
\int_{\mathbb{R}^{N}} \lambda_{\Omega}^{J} \tau(x) w(x) d x \leq \mathcal{F}_{J}(w)
$$

Now, since

$$
P_{J}\left(E_{t}(w) \backslash \Omega\right)=L_{J}\left(E_{t}(w) \backslash \Omega, \mathbb{R}^{N} \backslash\left(E_{t}(w) \backslash \Omega\right)\right)=L_{J}\left(E_{t}(w) \backslash \Omega,\left(E_{t}(w) \cap \Omega\right) \dot{\cup}\left(\mathbb{R}^{N} \backslash E_{t}(w)\right)\right),
$$


we have

$$
\begin{aligned}
I= & -\int_{-\infty}^{+\infty} P_{J}\left(E_{t}(w) \backslash \Omega\right) d t+\int_{-\infty}^{+\infty} 2 L_{J}\left(E_{t}(w) \backslash \Omega, E_{t}(w) \cap \Omega\right) d t+\lambda_{\Omega}^{J} \int_{-\infty}^{+\infty} \int_{E_{t}(w) \backslash \Omega} \tau(x) d x d t \\
=- & \int_{-\infty}^{+\infty} L_{J}\left(E_{t}(w) \backslash \Omega, \mathbb{R}^{N} \backslash E_{t}(w)\right) d t+\int_{-\infty}^{+\infty} L_{J}\left(E_{t}(w) \backslash \Omega, E_{t}(w) \cap \Omega\right) d t \\
& +\lambda_{\Omega}^{J} \int_{-\infty}^{+\infty} \int_{E_{t}(w) \backslash \Omega} \tau(x) d x d t \\
= & \int_{-\infty}^{+\infty}\left(\int_{E_{t}(w) \backslash \Omega}\left(\int_{\mathbb{R}^{N} \backslash E_{t}(w)}-J(x-y) d y+\int_{E_{t}(w) \cap \Omega} J(x-y) d y+\lambda_{\Omega}^{J} \tau(x)\right) d x\right) d t \\
= & \int_{-\infty}^{+\infty}\left(\int_{E_{t}(w) \backslash \Omega}\left(\int_{\mathbb{R}^{N} \backslash E_{t}(w)}(-\tilde{\mathbf{g}}(x, y)-1) J(x-y) d y\right) d x\right) d t \\
& +\int_{-\infty}^{+\infty}\left(\int_{E_{t}(w) \backslash \Omega}\left(\int_{E_{t}(w) \cap \Omega}(-\tilde{\mathbf{g}}(x, y)+1) J(x-y) d y\right) d x\right) d t \\
& -\int_{-\infty}^{+\infty}\left(\int_{E_{t}(w) \backslash \Omega}\left(\int_{E_{t}(w) \backslash \Omega} J(x-y) \tilde{\mathbf{g}}(x, y) d y\right) d x\right) d t .
\end{aligned}
$$

Now, the first integral is negative since $\tilde{\mathbf{g}}(x, y) \geq-1$ for $x \in \mathbb{R}^{N} \backslash \Omega$, the second is zero since $\tilde{\mathbf{g}}(x, y)=1$ for $x \in \mathbb{R}^{N} \backslash \Omega$ and $y \in \Omega$, and the last integral is zero since $\tilde{\mathbf{g}}=0$ in $\left(\mathbb{R}^{N} \backslash \Omega\right) \times\left(\mathbb{R}^{N} \backslash \Omega\right)$. Therefore, $I \leq 0$, and consequently (6.7) holds. Now (6.7) implies that (6.6) is true. Then, by Theorem 5.4, we have $-\lambda_{\Omega}^{J} \tau \in \Delta_{1}^{J}(0)$. Thus, there exists $\mathbf{g} \in L^{\infty}\left(\mathbb{R}^{N} \times \mathbb{R}^{N}\right), \mathbf{g}(x, y)=-\mathbf{g}(y, x)$ for almost all $(x, y) \in \mathbb{R}^{N} \times \mathbb{R}^{N}$, $\|\mathbf{g}\|_{\infty} \leq 1$, satisfying

$$
\int_{\mathbb{R}^{N}} J(x-y) \mathbf{g}(x, y) d y=-\lambda_{\Omega}^{J} \tau(x) \quad \text { a.e } x \in \mathbb{R}^{N} .
$$

and

$$
J(x-y) \mathbf{g}(x, y) \in J(x-y) \operatorname{sign}(0) \quad \text { a.e. }(x, y) \in \mathbb{R}^{N} \times \mathbb{R}^{N} .
$$

Now, multiplying by $\chi_{\Omega}$ and integrating by parts we get

$$
\begin{gathered}
\lambda_{\Omega}^{J}|\Omega|=\lambda_{\Omega}^{J} \int_{\mathbb{R}^{N}} \tau(x) \chi_{\Omega}(x) d x=-\int_{\mathbb{R}^{N}} \int_{\mathbb{R}^{N}} J(x-y) \mathbf{g}(x, y) \chi_{\Omega}(x) d x d y \\
\quad=\frac{1}{2} \int_{\mathbb{R}^{N}} \int_{\mathbb{R}^{N}} J(x-y) \mathbf{g}(x, y)\left(\chi_{\Omega}(y)-\chi_{\Omega}(x)\right) d x d y \leq P_{J}(\Omega)=\lambda_{\Omega}^{J}|\Omega|,
\end{gathered}
$$

from where it follows that

$$
J(x-y) \mathbf{g}(x, y) \in J(x-y) \operatorname{sign}\left(\chi_{\Omega}(y)-\chi_{\Omega}(x)\right) \quad \text { a.e. }(x, y) \in \mathbb{R}^{N} \times \mathbb{R}^{N},
$$

and consequently,

$$
-\lambda_{\Omega}^{J} \tau \in \Delta_{1}^{J} \chi_{\Omega} \quad \text { in } \mathbb{R}^{N}
$$

We now give a result that says that $J$-calibrability is related to the nonlocal curvature function $H_{\partial E}^{J}$, which is the nonlocal version of one implication in the equivalence between (a) and (c) in Theorem 6.7. 
Theorem 6.10. Let $\Omega \subset \mathbb{R}^{N}$ be a bounded set and assume $\int_{\Omega} J(x-y) d y>0$ for all $x \in \Omega$. Then,

$$
\Omega \text { is } J \text {-calibrable } \Rightarrow \underset{x \in \Omega}{\operatorname{ess} \sup } H_{\partial \Omega}^{J}(x) \leq \lambda_{\Omega}^{J} .
$$

Proof. By Theorem 6.9, there exists $\mathbf{g} \in L^{\infty}\left(\mathbb{R}^{N} \times \mathbb{R}^{N}\right)$ antisymmetric with $\|\mathbf{g}\|_{\infty} \leq 1$ and a function $\tau$, equal to 1 in $\Omega$, such that

$$
-\int_{\mathbb{R}^{N}} J(x-y) \mathbf{g}(x, y) d y=\lambda_{\Omega}^{J} \tau(x) \quad \text { a.e } x \in \mathbb{R}^{N},
$$

and

$$
J(x-y) \mathbf{g}(x, y) \in J(x-y) \operatorname{sign}\left(\left(\chi_{\Omega}(y)-\chi_{\Omega}(x)\right) \quad \text { a.e. in } \mathbb{R}^{N} \times \mathbb{R}^{N} .\right.
$$

Then, by (6.8), (6.9) and since $\tau=1$ in $\Omega$, we have

$$
H_{\partial \Omega}^{J}(x)=\int_{\mathbb{R}^{N}} J(x-y)\left(\chi_{\mathbb{R}^{N} \backslash \Omega}(y)-\chi_{\Omega}(y)\right) d y \leq-\int_{\mathbb{R}^{N}} J(x-y) \mathbf{g}(x, y) d y \leq \lambda_{\Omega}^{J} \quad \text { for a.e. } x \in \Omega .
$$

The reverse of Thyeorem 6.10 is not true in general, that is, condition (6.10) does not imply $J-$ calibrability in general. We don't know if for convex sets it is sufficient. In the next remark we provide some examples of non- $J$-calibrable sets and its interplay with (6.10).

Remark 6.11. 1. Observe that $\underset{x \in \Omega}{\operatorname{ess} \sup } H_{\partial \Omega}^{J}(x) \leq \lambda_{\Omega}^{J}$ if and only if

$$
\frac{1}{|\Omega|} \int_{\Omega} \int_{\Omega} J(x-y) d y d x \leq 2 \underset{x \in \Omega}{\operatorname{essinf}} \int_{\Omega} J(x-y) d y .
$$

2. In general,

$$
\underset{x \in \Omega}{\operatorname{ess} \sup _{\partial \Omega}} H_{\partial \Omega}^{J}(x) \leq \lambda_{\Omega}^{J} \text { does not imply } \Omega \text { is } J \text {-calibrable. }
$$

In fact. Assume that $\operatorname{supp}(J) \subset B_{R}(0)$. Let $R_{1}=R_{2}+\epsilon$, and $x_{1}, x_{2} \in \mathbb{R}^{N}$ such that $\left\|x_{1}-x_{2}\right\|>$ $R_{1}+R_{2}+2 R$. Consider $\Omega:=B_{R_{1}}\left(x_{1}\right) \cup B_{R_{2}}\left(x_{2}\right)$. Then, by Proposition 2.2 and the Isoperimetrical Inequality, we have

Therefore, $\Omega$ is not $J$-calibrable. Now,

$$
\lambda_{\Omega}^{J}=\frac{P_{J}\left(B_{R_{1}}\left(x_{1}\right)\right)+P_{J}\left(B_{R_{2}}\left(x_{2}\right)\right)}{\left|B_{R_{1}}\left(x_{1}\right)\right|+\left|B_{R_{2}}\left(x_{2}\right)\right|}>\frac{P_{J}\left(B_{R_{1}}\left(x_{1}\right)\right)}{\left|B_{R_{1}}\left(x_{1}\right)\right|} .
$$

$$
\underset{x \in \Omega}{\operatorname{ess} \sup } H_{\partial \Omega}^{J}(x)=\underset{x \in \Omega}{\operatorname{ess} \sup _{2}} H_{\partial B_{R_{2}}\left(x_{2}\right)}^{J}(x) \leq \frac{P_{J}\left(B_{R_{2}}\left(x_{2}\right)\right)}{\left|B_{R_{2}}\left(x_{2}\right)\right|} \leq \lambda_{\Omega}^{J}
$$

if $\epsilon$ is small enough.

Note that in this example $\Omega$ is not connected. However, this fact is not relevant. We have that the nonlocal perimeter and the nonlocal curvature are continuous with respect to the set in terms of convergence in measure (if $E_{n} \rightarrow E$ in the sense that $\left|E_{n} \triangle E\right| \rightarrow 0$ then $P_{J}\left(E_{n}\right) \rightarrow P_{J}(E)$ and $H_{\partial E_{n}}^{J}(x) \rightarrow$ $\left.H_{\partial E}^{J}(x)\right)$. Then we only have to connect the two balls with a thin bridge to obtain an example of a connected domain such that

$$
\underset{x \in \Omega}{\operatorname{ess} \sup } H_{\partial \Omega}^{J}(x) \leq \lambda_{\Omega}^{J} \text { but } \Omega \text { is not } J \text {-calibrable. }
$$

3. Let $\Omega \subset \mathbb{R}^{N}$ be a bounded set and assume $\int_{\Omega} J(x-y) d y>0$ for all $x \in \Omega$. One can ask if

$$
\sup _{x \in \partial \Omega} H_{\partial \Omega}^{J}(x) \leq \lambda_{\Omega}^{J} .
$$


implies $J$-calibrability. The following example shows that, in general, this is not the case. For $N=1$, and for $J(z)=\frac{1}{2} \chi_{[-1,1]}(z)$ and

$$
\left.\Omega_{\epsilon}=(]-3,3[\backslash[-1,1]) \cup\right]-\epsilon, \epsilon[, \quad 0<\epsilon<1 / 2,
$$

one can check that

$$
\sup _{x \in \partial \Omega_{\epsilon}} H_{\partial \Omega_{\epsilon}}^{J}(x)=(1-3 \epsilon)^{+}<H_{\partial \Omega_{\epsilon}}^{J}(0)=1-2 \epsilon=\sup _{x \in \Omega} H_{\partial \Omega_{\epsilon}}^{J}(x),
$$

and that

$$
\lambda_{\Omega_{\epsilon}}^{J}=\frac{1+2 \epsilon-3 \epsilon^{2}}{4+2 \epsilon} .
$$

Then, for for $\epsilon<\sqrt{19}-4$,

$$
1-2 \epsilon>\lambda_{\Omega_{\epsilon}}^{J}
$$

and consequently $\Omega$ is not calibrable. Now, for $0.236 \simeq \sqrt{5}-2 \leq \epsilon<\sqrt{19}-4 \simeq 0.359$ it holds that,

$$
\sup _{x \in \partial \Omega_{\epsilon}} H_{\partial \Omega_{\epsilon}}^{J}(x) \leq \lambda_{\Omega_{\epsilon}}^{J}
$$

and consequently condition (6.11) is not enough for $J$-calibrability.

4. Let $J$ be a nonnegative radially nonincreasing function. It is easy to see that a large cube $]-L, L\left[{ }^{N}\right.$ is not $J$-calibrable, this is also true in the local case for any cube.

On the other hand, in the local case, the stadium given by the convex hull of two circles $(N=2)$,

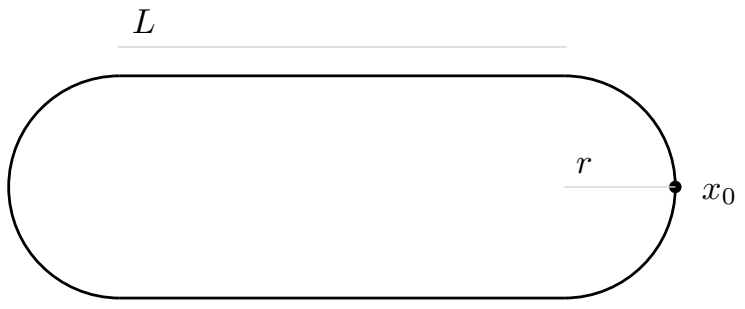

$$
\Omega_{r}^{L}:=\operatorname{co}\left(B_{r}\left(-\frac{L}{2}, 0\right) \cup B_{r}\left(\frac{L}{2}, 0\right)\right)
$$

with $L, r>0$, is calibrable. Indeed,

$$
\operatorname{ess}_{x \in \partial \Omega_{r}^{L}} H_{\partial \Omega_{r}^{L}}(x)=\frac{1}{r}<\frac{2 \pi r+2 L}{\pi r^{2}+2 r L}=\frac{\operatorname{Per}(\Omega)}{|\Omega|},
$$

and Theorem 6.7 gives the result. But $\Omega_{r}^{L}$ is not $J$-calibrable for $J=\frac{1}{\left|B_{1}(0,0)\right|} \chi_{\left.B_{1}(0,0)\right)}, r>1$ and $L>2$ large enough. Let us prove this fact. To this end, by Theorem 6.10 and the statement 1 of this remark, it is enough to show that, for $L$ large enough,

$$
\frac{1}{\left|\Omega_{r}^{L}\right|} \int_{\Omega_{r}^{L}} \int_{\Omega_{r}^{L}} J(x-y) d y d x>2 \underset{x \in \Omega_{r}^{L}}{\operatorname{essinf}} \int_{\Omega_{r}^{L}} J(x-y) d y .
$$

Now this condition reads as follows:

$$
\frac{1}{\left|\Omega_{r}^{L}\right|} \int_{\Omega_{r}^{L}}\left|\Omega_{r}^{L} \cap B_{1}(x)\right| d x>2\left|B_{1}\left(\frac{L}{2}+r, 0\right) \cap B_{r}\left(\frac{L}{2}, 0\right)\right|,
$$


since

$$
\underset{x \in \Omega_{r}^{L}}{\operatorname{essinf}} \int_{\Omega_{r}^{L}} J(x-y) d y=\int_{\Omega_{r}^{L}} J\left(x_{0}-y\right) d y
$$

for $x_{0}=\left(\frac{L}{2}+r, 0\right)$. Let us call $a(r):=2\left|B_{1}\left(\frac{L}{2}+r, 0\right) \cap B_{r}\left(\frac{L}{2}, 0\right)\right|$,

$$
a(r)=2\left(\arccos \left(\frac{1}{2 r}\right)+r^{2} \arccos \left(1-\frac{1}{2 r^{2}}\right)-\frac{1}{2} \sqrt{4 r^{2}-1}\right) .
$$

Now, if $\left.C_{r}^{L}=\right]-\frac{L}{2}, \frac{L}{2}[\times] 0, r-1\left[\right.$ and $\left.D_{r}^{L}=\right] 1-\frac{L}{2}, \frac{L}{2}-1[\times] r-1, r[$ we have, by symmetry,

$$
\begin{gathered}
\frac{1}{\left|\Omega_{r}^{L}\right|} \int_{\Omega_{r}^{L}}\left|\Omega_{r}^{L} \cap B_{1}(x)\right| d x \\
=\frac{1}{\pi r^{2}+2 r L} \int_{\Omega_{r}^{L}}\left|\Omega_{r}^{L} \cap B_{1}(x)\right| d x \geq \frac{2}{\pi r^{2}+2 r L} \int_{C_{r}^{L} \cup D_{r}^{L}}\left|\Omega_{r}^{L} \cap B_{1}(x)\right| d x \\
=\frac{2}{\pi r^{2}+2 r L}\left(\pi L(r-1)+\int_{D_{r}^{L}}\left|\Omega_{r}^{L} \cap B_{1}(x)\right| d x\right) .
\end{gathered}
$$

Moreover,

$$
\int_{D_{r}^{L}}\left|\Omega_{r}^{L} \cap B_{1}(x)\right| d x=(L-2) \int_{r-1}^{r}\left(\pi-\beta_{s}\right) d s
$$

where $\beta_{s}$ is the circular segment of height $h=1-(r-s)$ of the circle of radius 1 , that is,

$$
\beta_{s}=\arccos (r-s)-(r-s) \sqrt{1-(r-s)^{2}} \text {. }
$$

Hence,

$$
\int_{D_{r}^{L}}\left|\Omega_{r}^{L} \cap B_{1}(x)\right| d x=(L-2) \int_{r-1}^{r}\left(\pi-\arccos (r-s)+(r-s) \sqrt{1-(r-s)^{2}}\right) d s=(L-2)\left(\pi-\frac{2}{3}\right) .
$$

Therefore, we obtain

if

$$
\begin{aligned}
& \frac{1}{\left|\Omega_{r}^{L}\right|} \int_{\Omega_{r}^{L}}\left|\Omega_{r}^{L} \cap B_{1}(x)\right| d x \geq \frac{2}{\pi r^{2}+2 r L}\left(\pi L(r-1)+(L-2)\left(\pi-\frac{2}{3}\right)\right) \\
& \quad=\frac{2}{\pi r^{2}+2 r L}\left(\left(\pi r-\frac{2}{3}\right) L-\left(\pi-\frac{2}{3}\right) 2\right)>a(r)
\end{aligned}
$$

$$
L>\frac{\frac{1}{2} a(r) \pi r^{2}+\pi-\frac{2}{3}}{r\left(\pi-\frac{2}{3 r}-a(r)\right)}
$$

since $\pi-\frac{2}{3 r}>a(r)$. That is, we get that (6.12) holds true for $L$ large enough. Consequently, $\Omega_{r}^{L}$ is not $J$-calibrable.

5. Let us see that the non $J$-calibrable set $\Omega_{r}^{L}$ does not contain a $J$-Cheeger set. Arguing by contradiction, assume that there exists $E$ a $J$-Cheeger set of $\Omega_{r}^{L}$. Then, since $E$ is $J$-calibrable and having in mind the calculation made in $\mathbf{4}$, we have

$$
\begin{aligned}
a(r) & =\frac{2}{\pi}\left|B_{1}\left(\frac{L}{2}+r, 0\right) \cap B_{r}\left(\frac{L}{2}, 0\right)\right|<\frac{1}{\left|\Omega_{r}^{L}\right|} \int_{\Omega_{r}^{L}} \int_{\Omega_{r}^{L}} J(x-y) d y d x \\
& <\frac{1}{|E|} \int_{E} \int_{E} J(x-y) d y d x \leq 2 \inf _{x \in E} \int_{E} J(x-y) d y=\frac{2}{\pi} \inf _{x \in E}\left|B_{1}(x) \cap E\right| .
\end{aligned}
$$

On the other hand, consider a ball $B_{s}$ such that $\left|B_{s}\right|=|E|$. By the Isoperimetric Inequality and since $B_{s}$ is $J$-calibrable, we have

$$
a(r)<\frac{1}{|E|} \int_{E} \int_{E} J(x-y) d y d x \leq \frac{1}{\left|B_{s}\right|} \int_{B_{s}} \int_{B_{s}} J(x-y) d y d x \leq a(s),
$$


from where it follows that $s>r$, and consequently $E \nsubseteq B_{r}\left(-\frac{L}{2}, 0\right)$. Hence, if we define

$$
l^{+}:=\sup \left\{l \in\left[-\frac{L}{2}, \frac{L}{2}\right]:\left|B_{r}(l, 0) \cap E\right|>0\right\},
$$

we have $l^{+}>-\frac{L}{2}$. Therefore, for $-\frac{L}{2}<l<l^{+}$, we have

$a(r)<\frac{2}{\pi} \underset{x \in E}{\operatorname{essinf}}\left|B_{1}(x) \cap E\right| \leq \frac{2}{\pi} \operatorname{essinf}\left\{\left|B_{1}(x) \cap E\right|: x \in E \cap\left(B_{r}\left(l^{+}, 0\right) \backslash B_{r}(l, 0)\right)\right\} \leq a(r)+o\left(l^{+}-l\right)$.

Then, letting $l \rightarrow l^{+}$we arive to a contradiction.

Our last aim is to relate local and nonlocal Cheeger constants and local and nonlocal calibrable sets under rescaling. We will use the following result by A. Ponce (see also [17]) in the line of Theorem 2.9, by J. Dávila, given above.

Theorem 6.12 (Ponce [32]). Let $B \subset \mathbb{R}^{N}$ be open, bounded with a Lipschitz boundary, and let $0 \leq \rho_{\epsilon}$ radial functions satisfying (2.9). Let $\epsilon_{n} \downarrow 0$ as $n \rightarrow+\infty$. If $\left\{u_{n}\right\}_{n} \subset L^{1}(\Omega)$ is a bounded sequence such that

$$
\int_{B} \int_{B} \frac{\left|u_{n}(x)-u_{n}(y)\right|}{|x-y|} \rho_{\epsilon_{n}}(x-y) d x d y \leq K_{1, N} M,
$$

where $K_{1, N}$ is given in (2.10) and $M$ is a constant, then $\left\{u_{n}\right\}_{n}$ is relatively compact in $L^{1}(B)$. Moreover, if $u_{n_{j}} \rightarrow u$ in $L^{1}(B)$ then $u \in B V(B)$ and

$$
\int_{B}|D u| \leq M
$$

Proposition 6.13. Let $\Omega$ be an open bounded set of $\mathbb{R}^{N}$, then

$$
\lim _{\epsilon \downarrow 0} \frac{C_{J}}{\epsilon} h_{1}^{J_{\epsilon}}(\Omega)=h_{1}(\Omega) .
$$

Proof. Given $\delta>0$, there exists $E_{\delta} \subset \Omega$ such that

$$
h_{1}(\Omega)+\delta \geq \frac{\operatorname{Per}\left(E_{\delta}\right)}{\left|E_{\delta}\right|} .
$$

Then, by Theorem 2.10, we have

$$
h_{1}(\Omega)+\delta \geq \lim _{\epsilon \downarrow 0} \frac{C_{J}}{\epsilon} \frac{P_{J_{\epsilon}}\left(E_{\delta}\right)}{\left|E_{\delta}\right|} \geq \limsup _{\epsilon \downarrow 0} \frac{C_{J}}{\epsilon} h_{1}^{J_{\epsilon}}(\Omega) .
$$

By the arbitrariness of $\delta$, we get

$$
\limsup _{\epsilon \downarrow 0} \frac{C_{J}}{\epsilon} h_{1}^{J_{\epsilon}}(\Omega) \leq h_{1}(\Omega) .
$$

Let us now suppose that

$$
\liminf _{\epsilon \downarrow 0} \frac{C_{J}}{\epsilon} h_{1}^{J_{\epsilon}}(\Omega)<h_{1}(\Omega) .
$$

By (6.2), given $\epsilon>0$, there exists $u_{\epsilon} \in B V_{J_{\epsilon}}(\Omega), u_{\epsilon}=0$ in $\mathbb{R}^{N} \backslash \Omega,\left\|u_{\epsilon}\right\|=1$, such that

$$
h_{1}^{J_{\epsilon}}(\Omega) \leq \mathcal{F}_{J_{\epsilon}}\left(u_{\epsilon}\right) \leq h_{1}^{J_{\epsilon}}(\Omega)+\epsilon^{2} .
$$

Then, by (6.13),

$$
\liminf _{\epsilon \downarrow 0} \frac{C_{J}}{\epsilon} \mathcal{F}_{J_{\epsilon}}\left(u_{\epsilon}\right)<h_{1}(\Omega) .
$$


Therefore, there exists a sequence $\epsilon_{n}$ decreasing ot 0 such that

$$
\frac{C_{J}}{\epsilon_{n}} \mathcal{F}_{J_{\epsilon_{n}}}\left(u_{\epsilon_{n}}\right)<h_{1}(\Omega) \text {. }
$$

Then, for a large ball $B$ containing $\Omega$,

$$
\frac{C_{J}}{\epsilon_{n}} \mathcal{F}_{J_{\epsilon_{n}}}\left(u_{\epsilon_{n}}\right)=\frac{1}{K_{1, N}} \int_{B} \int_{B} \frac{\left|u_{\epsilon_{n}}(x)-u_{\epsilon_{n}}(y)\right|}{|x-y|} \rho_{\epsilon_{n}}(x-y) d x d y<h_{1}(\Omega),
$$

where

$$
\rho_{\epsilon}(z)=\frac{1}{2} C_{J} K_{1, N} \frac{|z|}{\epsilon} J_{\epsilon}(z) .
$$

Consequently, by Theorem 6.12 (in the proof of Theorem 2.10 we see that $\rho_{\epsilon}$ satisty the hypothesis in the theorem), we have that there exists a subsequence of $\epsilon_{n}$, denoted equal, such that

$$
u_{\epsilon_{n}} \rightarrow u \text { in } L^{1}(B),
$$

$u \in B V(B)$ and, since moreover $u=u \chi_{\Omega}$,

$$
\int_{B}|D u|=\int_{\Omega}|D u|+\int_{\partial \Omega}|u| d \mathcal{H}^{N-1}<h_{1}(\Omega) .
$$

But we also get that $\|u\|_{L^{1}(\Omega)}=1$ and consequently, from the above inequality, we get $\lambda_{1}(\Omega)<h_{1}(\Omega)$, which is a contradiction. Therefore, what we supposed in (6.13) is false and then

$$
\liminf _{\epsilon \downarrow 0} \frac{C_{J}}{\epsilon} h_{1}^{J_{\epsilon}}(\Omega) \geq h_{1}(\Omega),
$$

and the proof concludes.

Corollary 6.14. Let $\Omega$ be an open bounded set of $\mathbb{R}^{N}$. If $\Omega$ is $J_{\epsilon_{n}}$-calibrable for a sequence $\epsilon_{n} \rightarrow 0$ as $n \rightarrow+\infty$ then $\Omega$ is calibrable.

Proof. Since $\Omega$ is $J_{\epsilon_{n}}$-calibrable we have

$$
\frac{C_{J}}{\epsilon_{n}} h_{1}^{J_{\epsilon_{n}}}(\Omega)=\frac{C_{J}}{\epsilon_{n}} \frac{P_{J_{\epsilon_{n}}}(\Omega)}{|\Omega|} .
$$

Hence, by Theorem 2.10,

$$
\frac{C_{J}}{\epsilon_{n}} h_{1}^{J_{\epsilon_{n}}}(\Omega) \rightarrow \frac{\operatorname{Per}(\Omega)}{|\Omega|} \text { as } n \rightarrow+\infty .
$$

Then, by Proposition 6.13, we conclude that

$$
\frac{\operatorname{Per}(\Omega)}{|\Omega|}=h_{1}(\Omega),
$$

and consequently $\Omega$ is calibrable

Acknowledgment. JMM and JT are supported by the Spanish project MTM2012-31103, and JDR is partially supported by the Spanish project MTM2010-18128. 


\section{REFERENCES}

[1] N. Abatangelo and E. Valdinoci, A notion of nonlocal curvature. Numer. Funct. Anal. Optimiz. 35, 7-9, (2014), 793-815.

[2] F. Alter, V. Caselles and A. Chambolle, A characterization of convex calibrable sets in $\mathbb{R}^{N}$. Math. Ann. 332 (2005), 329-366.

[3] F. Alter and V. Caselles, Uniqueness of the Cheeger set of a convex body. Nonlinear Analysis TMA 70 (2009), 32-44.

[4] L. Ambrosio, Minimizing movements. Rend. Accad. Naz. Sci. XL Mem. Mat. Appl. (5) 19 (1995), 191-246.

[5] L. Ambrosio, N. Fusco and D. Pallara, Functions of Bounded Variation and Free Discontinuity Problems. Oxford Mathematical Monographs, 2000.

[6] L. Ambrosio, G. De Philippis, and L. Martinazzi, Gamma-convergence of nonlocal perimeter functionals. Manuscripta Math. 134 (2011), 377-403.

[7] F. Andreu, V. Caselles, and J.M. Mazón, Parabolic Quasilinear Equations Minimizing Linear Growth Functionals. Progress in Mathematics, vol. 223, 2004. Birkhauser.

[8] F. Andreu, J. M. Mazón, J. D. Rossi and J. Toledo, A nonlocal p-Laplacian evolution equation with nonhomogeneous Dirichlet boundary conditions. SIAM J. Math. Anal. 40 (2008/09), 1815-1851.

[9] F. Andreu, J. M. Mazón, J. D. Rossi and J. Toledo, A nonlocal p-Laplacian evolution equation with Neumann boundary conditions. J. Math. Pures Appl. 90 (2008), 201-227.

[10] F. Andreu, J. M. Mazón, J. D. Rossi and J. Toledo, The limit as $p \rightarrow \infty$ in a nonlocal p-Laplacian evolution equation: a nonlocal approximation of a model for sandpiles. Cal. Var 35 (2009), 279-316.

[11] F. Andreu-Vaillo, J. M. Mazón, J. D. Rossi and J. Toledo, Nonlocal Diffusion Problems. Mathematical Surveys and Monographs, vol. 165. AMS, 2010.

[12] J-F. Aujol, G. Gilboa and N. Papadakis, Fundamentals of Non-local Total Variation Spectral Theory. Proc. Scale and Variational Methods in Computer Vision (SSVM), 2015, pp. 66-77.

[13] Ph. Bénilan and M. G. Crandall, Completely Accretive Operators. In Semigroups Theory and Evolution Equations (Delft, 1989), Ph. Clement et al. editors, volume 135 of Lecture Notes in Pure and Appl. Math., Marcel Dekker, New York, 1991, pp. 41-75.

[14] L. Brasco, E. Lindgren and E. Parini, The fractional Cheeger problem. Interfaces Free Bound. 16 (2014), $419-458$.

[15] H. Brezis, Operateurs Maximaux Monotones. North Holland, Amsterdam, 1973.

[16] H. Brezis, Functional Analysis, Sobolev Spaces and Partial Differential equations. Universitext, Springer, 2011.

[17] J. Bourgain, H. Brezis and P. Mironescu, Another look at Sobolev spaces. In: Menaldi, J. L. et al. (eds.), Optimal control and Partial Differential Equations. A volume in honour of A. Bensoussan's 60th birthday, pages 439-455, IOS Press, 2001.

[18] L. Caffarelli, J.M. Roquejoffre and O. Savin, Nonlocal minimal surfaces. Comm. Pure Appl. Math. 63 (2010), 11111144 .

[19] L. Caffarelli and P.E. Souganidis, Convergence of nonlocal threshold dynamics approximations to front propagation. Arch. Ration. Mech. Anal. 195 (2010), 1-23.

[20] L. Cafarelli and E. Valdinoci, Uniform estimates and limiting arguments for nonlocal minimal surfaces. Calc. Var. Partial Differential Equations 41 (2011), 203-240.

[21] J. Dávila, On an open question about functions of bounded variation. Calc. Var. Partial Differential Equations 15 (2002), 519-527.

[22] I. Ekeland, Convexity methods in Hamiltonian mechanics. Springer-Verlag, Berlin, 1990.

[23] V. Fridman, B. Kawohl, Isoperimetric estimates for the first eigenvalue of the p-Laplace operator and the Cheeger constant. Comment. Math. Univ. Carolinae 44 (2003), 659-667.

[24] G. Franzina and E. Valdinoci, Geometric analysis of fractional phase transition interfaces. In Geometric Properties for Parabolic and Elliptic PDEs, Springer INdAM Series Volume 2, 2013, 117-130.

[25] D. Grieser, The first eigenvalue of the Laplacian, isoperimetric constants, and the max ow min cut theorem. Arch. Math. (Basel) 87 (2006), 75-85.

[26] G. Gilboa and S. Osher, Nonlocal operators with applications to image processing. SIAM Multiscale Model. Simul., 7 (2008), 1005-1028.

[27] E. Giusti, Minimal surface and functions of bounded variation. Monographs in Mathematics 80. Birkhäuser Verlag, Basel, 1984.

[28] G. H. Hardy, J. E. Littlewood, and G. Polya, Inequalities. Cambridge University Press, 1952.

[29] C. Imbert, Level set approach for fractional mean curvature flows. Interface Free Bound.11 (2009), 153-176.

[30] E. H. Lieb and M. Loss, Analysis. AMS Graduate Studies in Mathematics, Vol. 14. 1987. 
[31] A. Ponce, A new approach to Sobolev spaces and connections to $\Gamma$-convergence. Calc. Var. Partial Differential Equations, 19 (2004), 229-255.

[32] A. Ponce, An estimate in the spirit of Poincare's inequality. J. Eur. Math. Soc., 6 (2004), 1-15.

[33] O. Savin, E. Valdinoci, Regularity of nonlocal minimal cones in dimension 2. Calc. Var. Partial Differential Equations 48 (2013), 33-39.

[34] E. Valdinoci, A Fractional Framework for perimeter and Phase Transitions. Milan J. Math. 81 (2013), 1-23.

[35] A. Visintin, Nonconvex functionals related to multiphase systems. SIAM J. Math. Anal. 21 (1990), 1281-1304.

[36] A. Visintin, Generalized coarea formula and fractal sets. Japan J. Indust. Appl. Math. 8 (1991), $175-201$.

J. M. Mazón: Departament d’AnÀlisi Matemàtica, Universitat de València, Dr. Moliner 50, 46100 Burjassot, SPAIN. mazon@uv.es

J. D. Rossi: Departamento de Matemática, FCEyn UBA, Ciudad Universitaria, Pab 1 (1428), Buenos Aires, Argentina. jrossi@dm.uba.ar

J. Toledo: Departament d'Anàlisi Matemàtica, Universitat de València, Dr. Moliner 50, 46100 Burjassot, SPAIN. toledojj@uv.es 